\title{
Vehicle Assignment considering Battery Endurance for Electric Vehicle Carsharing Systems
}

\author{
Bo Zhang $\mathbb{D},{ }^{1}$ Lei Wang $\mathbb{D},{ }^{2}$ Li Li $\mathbb{D}^{1},{ }^{1}$ Xu Lei $\mathbb{D},{ }^{1}$ and Yongfeng Ju $\mathbb{D}^{1}$ \\ ${ }^{1}$ School of Electronics and Control Engineering, Chang'an University, Xi'an 710064, Shaanxi, China \\ ${ }^{2}$ College of Transportation Engineering, Tongji University, Shanghai 201804, China \\ Correspondence should be addressed to Lei Wang; wangleicuail@gmail.com
}

Received 10 March 2020; Revised 14 November 2020; Accepted 11 January 2021; Published 25 January 2021

Academic Editor: Rocío de Oña

Copyright (c) 2021 Bo Zhang et al. This is an open access article distributed under the Creative Commons Attribution License, which permits unrestricted use, distribution, and reproduction in any medium, provided the original work is properly cited.

On-demand station-based one-way carsharing is widely adopted for battery electric vehicle sharing systems, which is regarded as a supplement of urban mobility and a promising approach to the utilization of green energy vehicles. The service model of these carsharing systems allows users to select vehicles based on their own judgment on vehicle battery endurance, while users tend to pick up vehicles with the longest endurance distances. This phenomenon makes instant-access systems lose efficiency on matching available vehicles with diverse user requests and limits carsharing systems for higher capacity. We proposed a vehicle assignment method to allocate vehicles to users that maximize the utility of battery, which requires the system to enable short-term reservation rather than instant access. The methodology is developed from an agent-based discrete event simulation framework with a firstcome-first-serve logic module for instant access mode and a resource matching optimization module for short-term reservation mode. Results show that the short-term reservation mode can at most serve $20 \%$ more users and create $47 \%$ more revenue than instant access mode under the scenario of this research. This paper also points out the equilibrium between satisfying more users by efficiently allocating vehicles and distracting users by disabling instant access and suggests that the reservation time could be 15 minutes.

\section{Introduction}

Carsharing has been regarded as one of the innovative urban shared-use transportation modes since the booming of shared economy and mobile Internet technology. Users are allowed to drive the shared vehicles across the carsharing networks and pay for their hourly rentals. With the awareness of green energy promotion among the public and automobile manufacturers, electric vehicles have penetrated into the carsharing market, which provides environmental benefits for urban mobility $[1,2]$. Limited battery endurance and time-consuming recharging process at the current stage make electric vehicles lose attractiveness compared with internal combustion engine vehicles on the private car sales market. However, the carsharing market provides a promising breeding ground for electric vehicles since carsharing organizations (CSOs) manage charging station networks and available vehicles, which set users free from concerns on recharging and maintenance by themselves. Many carsharing enterprises introduce electric vehicles into their fleets, e.g., ShareNow and Communauto, while some others operate electric fleet only, e.g., EVCARD [3] and GoFun in China.

The station-based one-way carsharing model is believed to be an appropriate form for electric vehicle carsharing systems (EVCSs, or E-carsharing). Charging piles are usually installed at carsharing stations and users are required to plug in the chargers after parking the cars. For instance, EVCARD operates large charging station networks and allows users to pick up at one station and then drop off at any other station with the charging device plugged in. EVCARD is also an ondemand carsharing service that users can instantly choose any available vehicles with different state of charge (SOC) at the station; i.e., the user can see the exact SOC and battery endurance (possible maximum distance to drive) of the vehicles idling at station from the smartphone APP and instantly select a vehicle without reservation in advance. 
The on-demand one-way EVCS presents flexibility that users are endowed with the decision right to choose vehicles according to the SOCs as well as their expected trip distances. However, the user's behavior on choosing vehicles limits the matching efficiency between the demand on expected travel distance and the supply of vehicle battery endurance. For instance, some of the users prefer to occupy vehicles with high SOCs even travel shortly, while users that need to drive for long distances could not be satisfied. To mitigate the unbalance of matching on expected travel distance and battery endurance, it is necessary to develop an optimized vehicle assignment mechanism. It is possible to improve vehicle utilization and overall profits for the CSOs by assigning vehicles to users more reasonably rather than choosing by themselves, which helps CSOs to satisfy more users. The demand-vehicle matching also impacts the vehicle floating pattern over the networks and consequently influences the strategy of vehicle relocation and fleet rebalancing. The study on matching mechanism between expected travel distance of user and battery endurance of vehicle provides a better understanding on EVCS operation and improves the precision of vehicle relocation.

This paper presents the study on the matching mechanism between expected travel distance and battery endurance and assigning vehicles for users, i.e., to appoint vehicles with appropriate endurance distances for users according to their requested travel distances, to improve vehicle utilization and user satisfaction. The remainder of the paper is organized as follows. Section 2 reviews the literature on carsharing operation and electricity management. Section 3 proposes the matching methods including first-come-firstserve (FCFS) and short-term optimization based on a simulation scheme. Section 4 provides a case study and presents the findings by introducing the matching methods. Section 5 concludes the study.

\section{Literature Review}

In this section, we first review the classifications of different carsharing systems and point out the orientation of the ondemand one-way EVCS. Subsequently, the electricity problems about electric vehicle operation and management have been summarized. Finally, we introduce problems and methods for vehicle relocation, fleet management, and operation improvement to seek the potential of user-vehicle assignment for improving the efficiency of EVCS.

2.1. Categorizing Carsharing Systems. Carsharing attracts attention around the globe and many carsharing enterprises have emerged since the last decade. New startups continue innovation on service models that present different characteristics and various degrees of flexibility.

At the very beginning stage of research towards carsharing since 2002, Barth and Shaheen originally summarized carsharing systems and categorized them into three models: (1) neighborhood carsharing, (2) station cars, and (3) multi-nodal shared-use vehicle [4]. Cepolina et al. [5] introduced new features for carsharing models including instant access, open-ended reservation, and one-way trip enabling and suggested that CSOs tended to provide better flexibility for users. Ferrero et al. developed a classification method and differentiated carsharing by dimensions such as mode, engine, objectives, time horizon, and methodologies. Remane et al. proposed a taxonomy for carsharing business models and extracted 43 characteristics in double layer and 15 dimensions to classify the carsharing business model. Wang et al. [6] also classified carsharing services into types of round-trip, one-way, station-based, free-floating, reservation-based, and on-demand, which also pointed out that the emerging EV-only carsharing CSOs tended to adopt the station-based on-demand model.

Based on the existing taxonomy and considering the requirement of this study, we summarize the categories of carsharing business in Table 1. A carsharing system presents several characteristics, and combinations of these characteristics shape various kinds of carsharing business, which provide different degrees of flexibility to users [7]. It should be noted that many of the newly launched EVCSs enabled one-way mode and are station-based for the convenience of installing recharging piles and maintaining vehicles, though some of them are free-floating for better flexibility with the sacrifice on vehicle relocation costs [8]. It should be noted that, according to the statistics of Remane et al. [9], 100\% of the one-way station-based systems are instant access without any reservation.

Since the one-way station-based on-demand battery electric vehicle carsharing systems play a vital role in the global carsharing business, in this paper, we study the vehicle assignment problem for users according to their desired travel distance under the schemes of instant access and short-term reservation and explore the possibility to improve the operation efficiency.

2.2. Electricity Problems in EVCS. The EVCS performs quite differently from other kinds of carsharing systems because of the limitation of battery endurance and recharging time. Model innovations are made including battery swapping [10] and the operation scheduling algorithm is proposed. Other types of vehicles are also discussed, such as plug-in [11] hybrid electric [12] vehicle charging problem and shared shuttle bus scheduling [13]. For EVCS that we discuss in this paper, the charging scheduling problem is also a big issue [14].

Many studies explored the electricity problems in EVCS from different perspectives, including the "range anxiety," charging station planning, and vehicle routing for charging devices. Hu et al. [15] investigated the effect of battery capacity on electric vehicle sharing systems and suggested that limited battery capacity lowers user satisfaction and vehicle utilization. $\mathrm{Hu}$ et al. also mentioned "range anxiety" and a vehicle selection principle [16] that allocates vehicles for users according to SOC for on-demand EVCS. Because the existence of anxiety, they assume that user must choose the vehicle with $\max$ SOC. The range anxiety does impact traveler behavior [17] and influence the vehicle floating pattern within networks [18]. 
TABLE 1: Categories of carsharing business models.

\begin{tabular}{|c|c|c|}
\hline Dimension & Type & Description \\
\hline Trip & $\begin{array}{c}\text { Round-trip } \\
\text { One-way }\end{array}$ & $\begin{array}{l}\text { Users rent cars from one place and must return to the same place } \\
\text { Users are allowed to pick up vehicles from one place and drop off at another place without } \\
\text { compulsorily returning to the original place, e.g., Car2Go and EVCARD }\end{array}$ \\
\hline $\begin{array}{l}\text { Service } \\
\text { range }\end{array}$ & Station-based & $\begin{array}{l}\text { Stations are designed in the networks and users should pick up and drop off only at stations, e.g., } \\
\text { EVCARD (round-trip should be station-based) } \\
\text { Service areas are designed and users are allowed to pick up and drop off at any legal parking place } \\
\text { within the service area without compulsorily driving to stations, e.g., Car2Go (free-floating can be } \\
\text { one-way, and one-way can be both station-based and free-floating) }\end{array}$ \\
\hline & & $\begin{array}{c}\text { Users should make reservations for vehicles by providing their trip information usually one day or } \\
\text { more before their trips }\end{array}$ \\
\hline Acce & $\begin{array}{l}\text { Short-term } \\
\text { reservation } \\
\text { Instant access/on- } \\
\text { demand }\end{array}$ & Users should make reservations for vehicles on several hours or minutes ahead of their trips \\
\hline Vehicle type & $\begin{array}{l}\text { Fuel vehicle } \\
\text { Battery electric } \\
\text { vehicle } \\
\text { Other green energy }\end{array}$ & $\begin{array}{c}\text { System operates vehicles equipped with internal combustion engines and can be refueled easily } \\
\text { System operates battery electric vehicles (BEVs) which have limited driving range and recharging } \\
\text { speed } \\
\text { System operates plug-in hybrid electric vehicles or other green energy vehicles }\end{array}$ \\
\hline
\end{tabular}

To mitigate the range anxiety problem, on the planning level, CSOs can optimize the location of charging stations in EVCS to cover more destinations as well as to save construction costs for not only E-carsharing systems $[19,20]$ but also general electric vehicle drivers [21]. From the user experience aspect, some studies proposed vehicle routing suggestions for users to optimize their routes for charging stations and to relieve their anxiety $[22,23]$.

For the vehicle assignment problem with battery consumption information requests, Zhang et al. proposed an optimization model considering multiple vehicle relays and could output the arrangements of vehicle utilization for users [24]. However, the overall optimization models of networks and event flows should assume all demands are preknown, which could be long-term reservation or precisely predicted. One-way CSOs do not prefer long-term reservation since it loses flexibility and on the other hand the precise prediction of demand is still difficult because carsharing systems are highly stochastic. In our study, we try to focus on the vehicle assignment or selection mechanism in the circumstances of short-term reservation or on-demand. To the best of our knowledge, there is less literature revealing the characteristics, battery matching, and vehicle assignment for short-term and on-demand systems and proposing improvement suggestions for them.

\subsection{Fleet Management and Operation Improvement.} Extensive studies have been conducted on fleet management, vehicle relocation, and operation decision support. Those works address core issues-balancing car distribution to meet the demand patterns-for sustainable, efficient, and effective carsharing operation. They typically fall into two folds: simulation [25-28] and optimization [29-32].

The optimization approach relies on operation research (OR) techniques to realize the objectives and on sophisticated graph or network modeling to reveal the system dynamics $[33,34]$. Solutions from OR present the optimum if precise demands are known. This requires system to be long-term reservation type, or strongly assume demand can be accurately and stably predicted $[35,36]$. Up to now, many studies are exploring demand prediction $[37,38]$ but still not quite accurate and robust enough to drive the optimization. Towards the instant access type, the system presents strong randomness and dynamics; simulation approaches perform well to model the system characteristics. To improve the performance, researchers also attached optimization to the simulation framework [39, 40]. Inventory control approaches [41-43] are also developed to cope with the dynamic fleet management tactics for instant access systems.

Note that some of the studies above consider fuel fleets rather than electric fleets. EVCS performs different characteristics on range anxiety and charging time, while users are not sensitive to fuel consumption and CSOs are not hindered by refueling for fuel vehicle carsharing systems. Taking electricity characteristics is quite important for EVCS modeling. For instance, the simulation framework of $\mathrm{Hu}$ et al. [15] adopted a maximum SOC pick-up strategy as we have discussed above. Wang et al. [42] formulated SOC constraints and integrated them in the overall optimization model, while the actual vehicle selection judged by users on SOCs would highly differ from the system optimization expectation. Ma et al. [43] involved a matching model between required distance and SOC under the objective of maximizing battery utilization, which is also not realistic enough to reveal vehicle selection by users in instant access systems or vehicle assignment in short-term reservation systems. In our paper, we attempt to explore the matching problem between user's desired travel distance and the vehicle's actual SOC, and to provide suggestions for vehicle assignment. This problem has another aspect of property which is the contrast between short-term reservation and the instant access system. The benefit of short-term reservation and the impacts to the experience of users should both be 
considered. Hence, this paper adopts an agent-based simulation and vehicle assignment optimization to model the short-term reservation and explores the natures of shortterm reservation and instant access.

\section{Methodology}

3.1. Agent-Based Simulation Framework. The methodology of this study is on the simulation basis with an optimization module for vehicle assignment. Agent-based simulation models are fundamental works to depict carsharing systems $[15,28]$. This paper further develops carsharing agent-based model by considering the vehicle assignment problem. We adopt agents to reveal the status of users and vehicles and introduce a matching mechanism to reveal the vehicle assignment. Agents are as follows.

(i) Users are generated with trip requests. User $i$ is defined by a tuple $u_{i}=\left(O_{i}, D_{i}, t_{0 i}, t_{1 i}, d_{i}, m_{i}, r_{i}, s_{i}\right)$, where $O_{i}$ denotes the origin station, $D_{i}$ denotes the destination station, $t_{0 i}$ denotes the departure time, $t_{1 i}$ denotes the arrival time, $d_{i}$ denotes the travel distance, $m_{i}$ is a $0-1$ sign that $m_{i}=1$ if user $i$ is matched with a vehicle that can satisfy the user's trip otherwise $=0, r_{i}$ denotes whether the user is occupying and driving a car on road and $r_{i}=1$ if driving otherwise $=0$, and $s_{i}$ denotes whether the user is served and $s_{i}=1$ if served otherwise $=0$. Note that we adopt simulation second $(s)$ to quantify time variables.

(ii) Vehicles transport users form one station to another. Vehicle $j$ is defined by a tuple $v_{j}=\left(e_{j}, p_{j}, t_{A j}, r_{O j}\right)$, where $e_{j}$ denotes the instant state of charge (SOC) of vehicle $j$ (defined by the current travel distance endurance, $\mathrm{km}), p_{j}$ denotes the position of $v_{j}$ by the latest station where $v_{j}$ is parking, $t_{A j}$ denotes the arrival time at $p_{j}$ and $t_{A j}=0$ for initialization, and $r_{O j}$ denotes the occupancy of the vehicle and $r_{O j}=1$ if $v_{j}$ is occupied by a user; otherwise $r_{O j}=0$.

Besides the agents, we define a matching between the user and vehicle:

(i) The matching between user and vehicle is defined as a tuple $m_{i j}=\left(u_{i}, v_{j}\right)$. This matching relates a user to a designated vehicle. Once the matching is decided, the users which have been transported and the service chain of vehicles can be speculated.

Note that these variables are time varying, and snapshots related to time instant $t$ as $u_{i}(t), v_{j}(t)$ and $m_{i j}(t)$ can be generated to record trajectories of users and vehicles. The process of the interaction between user agents and vehicle agents is described in Figure 1. Note that the states of vehicles and users change at discrete times and are updated by events; we adopt discrete event simulation (DES) to model the dynamics of the system.

3.2. User Interaction Modes Design. Considering that it has been reviewed that the existing one-way station-based systems are instant access [9] and the overall optimization relies on a strong assumption of long-term reservation or precise demand prediction [36], this research will focus on instant access mode and short-term reservation mode. The interaction modes are defined as follows.

(i) Instant access: First, users emerge and are allowed to inspect the SOCs of all available vehicles at the nearby station. A user will lock a car once the user makes a trip request and places an order. We assume the server system processes the requests one by one in a time sequence, i.e., first-come-first-serve (FCFS) (shown in Figure 2). Note that simultaneously arrived requests in reality will also be processed in a queue with a sequence of other properties like user number. In this simulation system, we generate users sequentially.

(ii) Short-term reservation: Users emerge in sequence and are grouped by time batch $\tau$. Users submit departure station, arrival station, and expected departure time to the system. For users whose departure stations are the same and departure times are in the same time batch, the system assigns available vehicles to those users according to some principles (shown in Figure 3).

3.3. Instant Access Mode. The instant access mode can be modeled as an agent-based simulation driven by discrete event simulation (DES) with asynchronous events. The events in this simulation can be found in Figure 4. Details of the events are defined in the following contents. An event list should be generated to ensure the sequence and action of each event. The event list can be dynamically read and written. Simulation progresses through the simulation seconds and triggers the event when processing it.

Event E1. User emerges. All users are generated in a set $U=\left\{u_{1}, u_{2}, \ldots, u_{i}, \ldots, u_{|U|}\right\}$ at the very beginning of simulation, where $|U|$ denotes the number of users in set $U . \forall u_{i}=\left(O_{i}, D_{i}, t_{0 i}, t_{1 i}, d_{i}, m_{i}, r_{i}, s_{i}\right) \in U$, suppose the request of user $i$ appears instantly at time $t_{0 i}$ and omit the reservation time, where $m_{i}=r_{i}=s_{i}=0$ for initialization.

Event E2. Vehicle arrives. Vehicles are in set $V$ and are grouped by two sets $V_{I}$ and $V_{R}$ for idling vehicles and running vehicles, respectively. First, considering station $n \in N$ where $N$ denotes the set of all stations and the number of stations is $|N|$, let the set of vehicles in station $n$ be $V_{n}$, and we have

$$
\begin{aligned}
& V_{I}=\underset{n \in N}{\cup} V_{n}, \\
& V_{n}=\left\{v_{j} \mid p_{j}=n \text { and } r_{O j}=0, \forall v_{j} \in V\right\} .
\end{aligned}
$$

For the running vehicles, there is

$$
V_{R}=V-V_{I} \text {. }
$$

Note that $V_{R}$ can be $\varnothing$ if all vehicles are at stations for initialization. For the running vehicles $\forall v_{j} \in V_{R}$, there must be a $m_{i j}$ relating $v_{j}$ to $u_{i}$ (the matching should be 


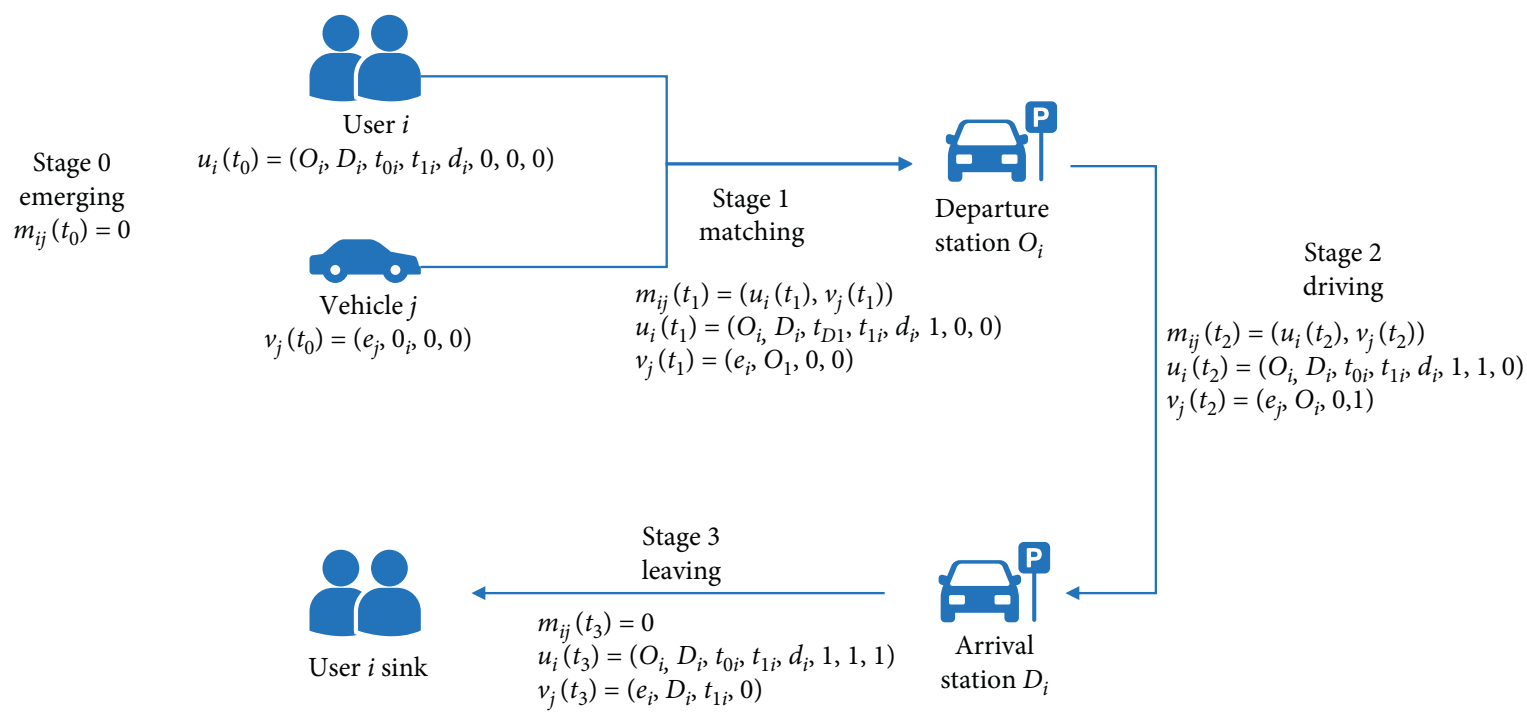

FIGURE 1: Interaction between user agent and vehicle agent.

ensured by event E3), and vehicle $j$ would arrive at station $D_{i}$ at $t_{1 i}$. When the arriving event happens at $t_{1 i}$ (i.e., E2 happens), update the following variables:

$$
\begin{aligned}
e_{j} & :=e_{j}-d_{i}, \\
p_{j} & =D_{i}, \\
t_{A j} & =t_{1 i}, \\
r_{O j} & =0, \\
V_{R} & :=V_{R}-\left\{v_{j}\right\}, \\
V_{D_{i}} & :=V_{D_{i}} \cup\left\{v_{j}\right\} .
\end{aligned}
$$

Event E3. User selects vehicle. $\forall u_{i} \in U$ this E3 event happens at $t_{0 i}$. When E3 happens, $\forall v_{j} \in V_{O_{i}}$, update the SOCs:

$$
e_{j}:=\min \left\{e_{j}+R \times\left(t_{0 i}-t_{A j}\right), e_{M}\right\}
$$

where $R$ denotes the charging speed $(\mathrm{km} / \mathrm{s})$ and $e_{M}$ is the maximum endurance distance of this type vehicle. Then matching will be processed as the following two schemes:

(a) Maximum SOC selection. Article [15] investigated user's choosing behavior and assumed all users would pick up the vehicle with maximum SOC. The maximum SOC selection can be expressed as follows:

$$
m_{i j}= \begin{cases}\left(u_{i}, v_{j}^{\left.\arg \max _{v_{j} \in V_{O_{i}}}\left(e_{j}\right)\right),}\right. & \max _{v_{j} \in V_{O_{i}}}\left(e_{j}\right) \geq d_{i}+\varepsilon \\ 0, & \max _{v_{j} \in V_{O_{i}}}\left(e_{j}\right)<d_{i}+\varepsilon\end{cases}
$$

where $\varepsilon$ denotes a SOC conservation in case that the vehicle is out of energy. (b) Random selection with range constraint. We also recognized some of the users would randomly choose from vehicles with enough power to reach their destinations. This mechanism is expressed as follows. For station $O_{i}$ and user $i$, the set of available vehicles is

$$
V_{A i}=\left\{v_{j} \mid e_{j} \geq d_{i}+\varepsilon, \forall v_{j} \in V_{O_{i}}\right\}
$$

The matching could be

$$
m_{i j}= \begin{cases}\left(u_{i}, \operatorname{rand}\left(V_{A i}\right)\right), & V_{A i} \neq \varnothing, \\ 0, & V_{A i}=\varnothing,\end{cases}
$$

where $\operatorname{rand}\left(V_{A i}\right)$ is a function which randomly returns a $v_{j}$ from $V_{A i}$.

We assume that users can be either maximum SOC selection or random selection, which follows a binary distribution of $p r$, where $p r$ is the probability that a user is maximum SOC selection. User $i$ falls into scheme (a) according to $p r$ and into scheme (b) according to $1-p r$.

After matching process if $m_{i j}=0$ then no action should be taken. Otherwise, the following variables should be updated for user $i$ and vehicle $j$ at time $t_{0 i}$ :

$$
\begin{aligned}
m_{i} & =1, \\
r_{O j} & =1, \\
V_{R} & :=V_{R} \cup\left\{v_{j}\right\}, \\
V_{O_{i}} & :=V_{O_{i}}-\left\{v_{j}\right\} .
\end{aligned}
$$

Event E4. This is where departure and transport take place. This event would happen at time $t_{0 i}+t_{M}$ after the matching is done, where $t_{M}$ denotes a very short time 


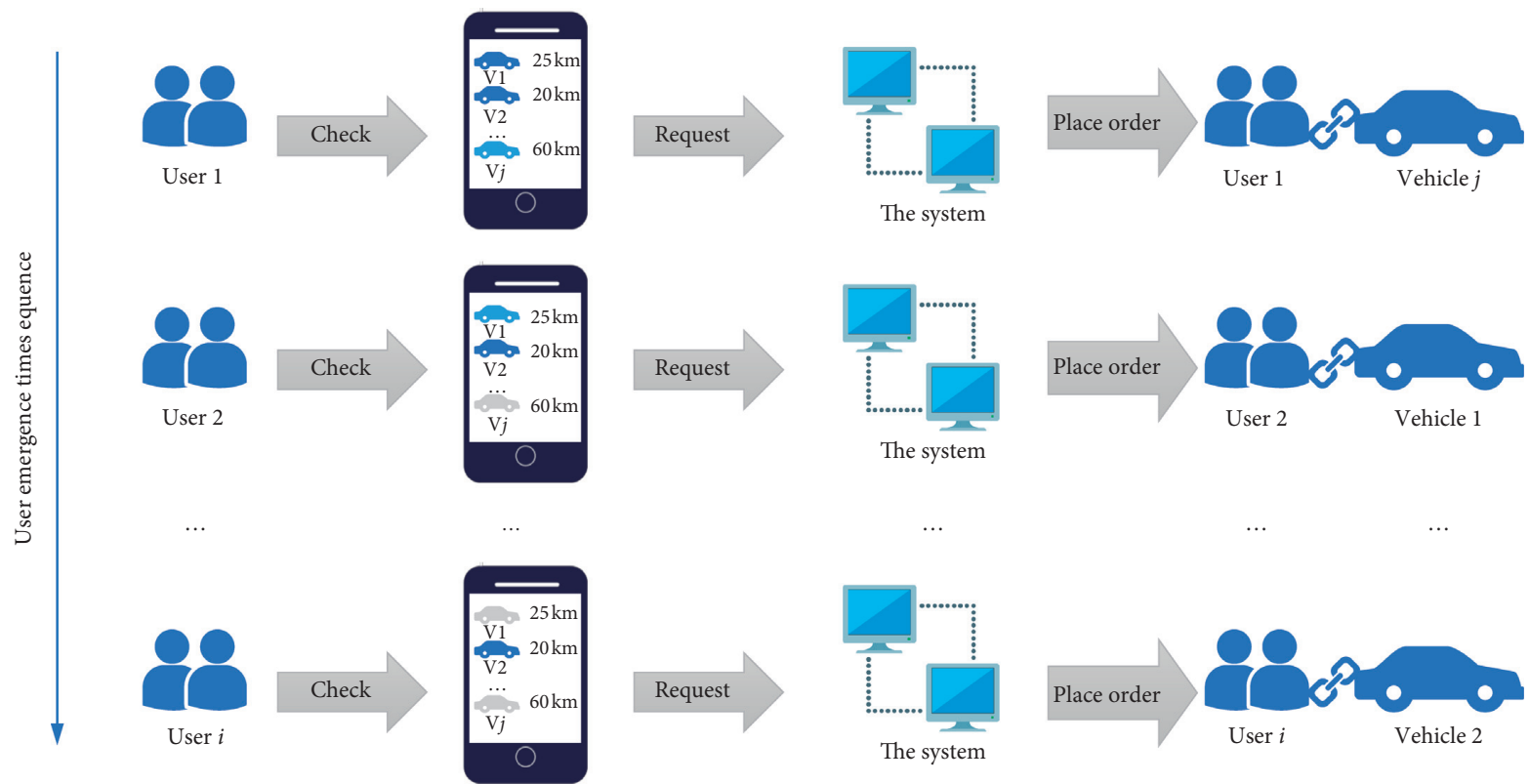

FIGURE 2: User interaction and vehicle selection of instant access mode.

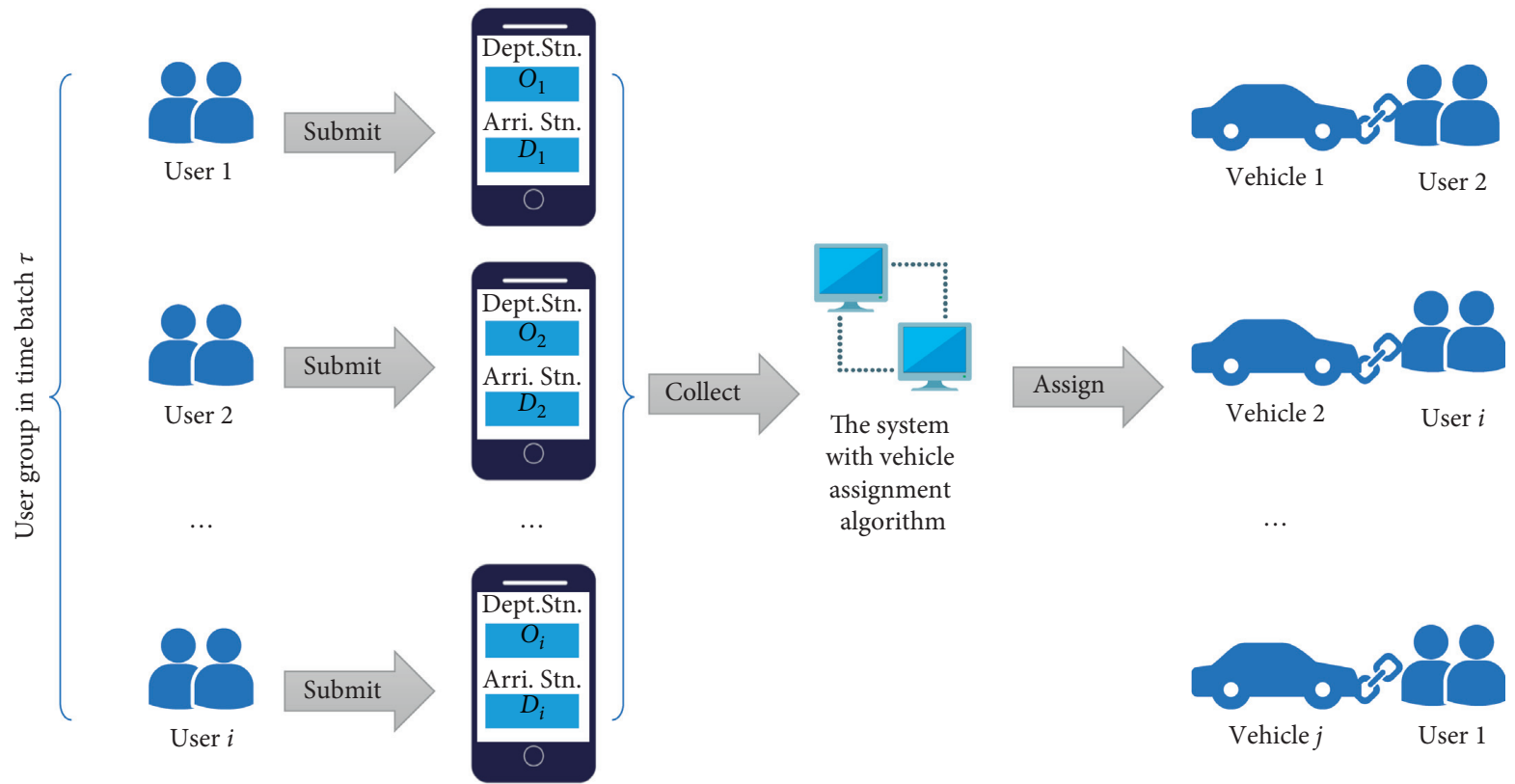

FIGURE 3: User interaction and vehicle assignment of short-term reservation mode.

interval. For user $i$ after activating event E3, we apply E4 to denote that the user is being transported.

$$
r_{i}= \begin{cases}1, & m_{i j} \neq 0, \\ 0, & m_{i j}=0 .\end{cases}
$$

Event E5. User sinks. This event eliminates the user if the user arrives at the destination. This event happens at $t_{1 i}$ if the user was transported.

$$
s_{i}= \begin{cases}1, & m_{i j} \neq 0, \\ 0, & m_{i j}=0 .\end{cases}
$$

Note that the users which cannot be served can be marked by $s_{i}=0$ and would not be processed again because $t_{1 i}$ in time sequential simulation would be processed only once.

3.4. Short-Term Reservation Mode. The FCFS instant access 


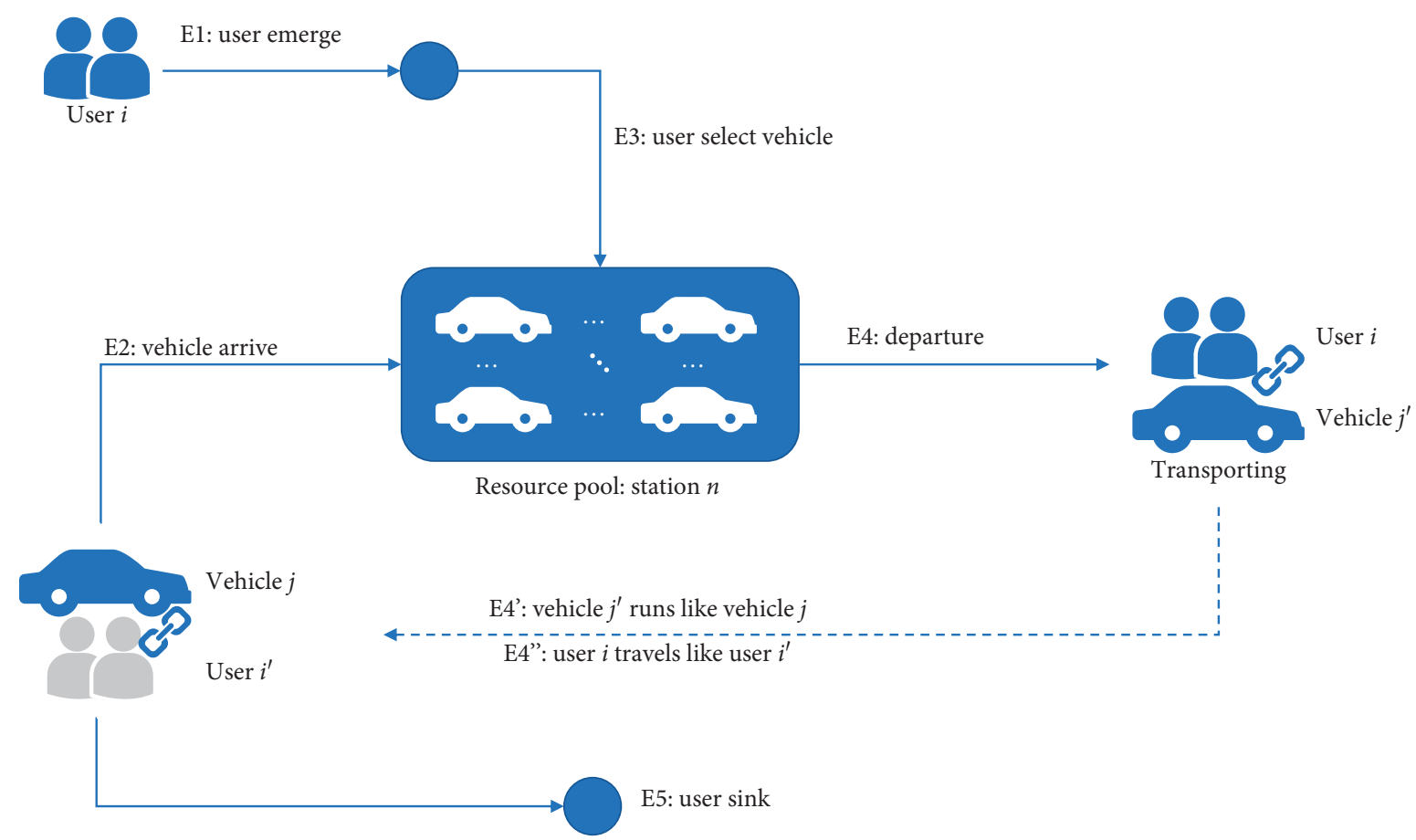

FIGURE 4: Events in instant access mode.

ystem loses optimality on matching between trip requests and vehicle supply, especially under circumstances that firstcoming users tend to occupy a vehicle with greater SOC. Consequently, the latter users with farther distance requests are more probable to be rejected, and vehicles with lower SOC may idle for a longer time without making profits.

We suggest that adding a matching process by gathering trip distance requests and allocating vehicles for users would increase efficiency. The matching process requires the system to be short-term reservation mode. This mode also enables dynamic vehicle relocation for satisfying more demands. To be focused, we concentrate on SOC matching and do not involve the impacts brought by vehicle relocation.

The methodology of short-term reservation mode follows the DES as well but is with a cyclic event of demand collection. The users are generated the same as instant access mode. Users are grouped by a cycle of $\tau$ in short-term reservation mode. Users' expected travel distances would be matched with the endurances of all vehicles. After matching, vehicles will be assigned to users. Users accepted by the system will drive the vehicle to the destinations and then exit the system. This process is driven by a cyclic event and several asynchronous events and can be described as in Figure 5.

Among the events of short-term reservation simulation, events F1, F2, F4, and F5 show similar properties to events E1, E2, E4, and E5, respectively, and will not further be explained. Note that C3 processes quite differently from E3 and is described as follows.

Event C3. Vehicle assignment based on battery utility maximization: Define $\tau$ (simulation seconds, s) as the time span to collect user requests. Starting from simulation 0 seconds, when it comes to $\tau s$, the first demand collection and vehicle assignment will be processed. Every $\tau s$, i.e., the $0 \mathrm{~s}, \tau \mathrm{s}, 2 \tau \mathrm{s}, 3 \tau \mathrm{s}, \ldots, k \tau \mathrm{s}, \ldots, K \tau \mathrm{s}$, the matching event is activated.

Users are initially generated the same as instant access mode. Note that user's departure time should be later than the time when the matching is processed. Hence, for the $k \tau s$ at station $n$, the users can be grouped in a set as

$$
U_{k n}=\left\{u_{i} \mid O_{i}=n \text { and } k \tau \leq t_{0 i} \leq(k+1) \tau, \forall u_{i} \in U, \forall k=0,1, \ldots, K\right\}
$$

For $V_{n}$, the SOCs should first be updated when C3 happens.

$$
e_{j}:=\min \left\{e_{j}+R \times\left(k \tau-t_{A j}\right), C_{M}\right\} .
$$

P1. Battery utility maximization. First, we define the concept of battery utility. If the system assigns $v_{j}$ to $u_{i}$, we define $d_{i} \cdot e_{j}$ as the battery utility of the match $m_{i j}$. To further explain it, we visualize an example of the matching in Figure 6(a). A user should at most be assigned to one vehicle, and a vehicle should at most be assigned to one user. One combination shown in this figure is that $u_{2}$ is assigned to $v_{1}$ and $u_{i}$ is assigned to $v_{2}$. We discover two possible ways to 
improve the matching. One is to serve more users; the other is to make full use of the available SOCs. They can be both captured by maximizing the total battery utility. The battery utility is visualized as the areas in Figure 6(b). If more users are matched with vehicles, the areas should be larger; if the SOC of vehicle better suits the required distance of user, the areas should also be larger (i.e., if assigning a vehicle with long distance to a user who travels nearby, the area would be small). Hence the objective is to maximize the total battery utility.

With $u_{i} \in U_{k n}$ and $v_{j} \in V_{n}$ at $k \tau$ known, the matching can be processed as a $0-1$ programming model:

$$
\begin{gathered}
\underset{x_{i j}}{\arg \max } \sum_{u_{i} \in U_{k n}} \sum_{v_{j} \in V_{n}} d_{i} \cdot e_{j} \cdot x_{i j}, \\
\text { s.t. } \sum_{u_{i} \in U_{k n}} x_{i j} \leq 1, \quad \forall v_{j} \in V_{n}, \\
\sum_{v_{j} \in V_{n}} x_{i j} \leq 1, \quad \forall u_{i} \in U_{k n}, \\
\left(e_{j}-d_{i}-\varepsilon\right) x_{i j} \geq 0, \quad \forall u_{i} \in U_{k n}, \forall v_{j} \in V_{n},
\end{gathered}
$$

where decision 0 - 1 variables $x_{i j}$ mark that the vehicle $j$ is assigned to user $i$ if $x_{i j}=1$; otherwise 0 ; objective (13) is to maximize the utility of battery range; i.e., if more vehicles matched users, and if the expected travel distance of user $i$ comes closer to the matched SOC of vehicle $j$, the objective value would be greater; constraint (15) ensures that one vehicle should at most be assigned to one user; constraint (16) ensures that one user should at most be served by one car; constraint (18) requires that the battery endurance of vehicle $j$ should be more than the required travel distance of user $i$ with a range conservation $\varepsilon$.

$P 2$. Battery utility and subsequent orders maximization. In this research, we doubt that though longrange users are more likely to be satisfied, the shortrange users also have opportunity to drive cars to a near station and following users would continue to rent this vehicle, which might not idle the system significantly, and could be a fact that the endurance range matching loses importance. Consequently, we propose a new principle to consider the potential subsequent trips and prefer to serve trips to "hot" stations that the vehicles would be utilized by latter users rather than be idled.

First, we define weight of station $n$ as the proportion of demands that would depart from it:

$$
W_{n}=\frac{\left|\left\{u_{i} \mid O_{i}=n, \forall u_{i} \in U\right\}\right|}{|U|}, \quad \forall n \in N .
$$

The objective is rewritten as

$$
\underset{x_{i j}}{\arg \max } \sum_{u_{i} \in U_{k n}} \sum_{v_{j} \in V_{n}} d_{i} \cdot e_{j} \cdot W_{D_{i}} \cdot x_{i j},
$$

s.t. (15)-(18).

It should be noted that short-term reservation mode presents less flexibility than instant access mode so that a portion of users may not turn to accept short-term reservation. It can be assumed that the probability that a user would deny to use carsharing relates to the reservation time $\tau$; i.e., we assume that a function like

$$
P=f(\tau),
$$

where $p s$ is the probability that users quit carsharing and $f$ is the function. $f$ can be an empirical function that returnsdifferent proportions of quited user if $\tau=1,5,15,30,60$ minutes. Users set $U$ should be changed to $U^{\prime}$ according to probability.

$$
U^{\prime}=\left\{u_{i} \mid \text { rand } \leq 1-P, \forall u_{i} \in U\right\}
$$

where rand denotes a random number between 0 and 1 .

\section{Case Study}

4.1. Data Description. This study is examined by adopting original transaction data from an EVCS company in Shanghai, China, which involves 56 stations and 2,847 transactions in a month. Note that those transactions represent served trips but not the input demands. To drive the simulation, we randomly generate input demands based on the original transaction data. First, we merge the transactions into one day, and we generate one-day trips from the original one-month data. Second, to avoid the bias of simulation samples, we generate 10 copies of the 2,847 transactions, where each copy records the transactions in a random sequence. Examples of the 10 simulation copies can be found in the online data repository. Third, we group the 2,847 transactions in each copy into four groups. Each group contains different quantity of transactions, which reflect different intensity of input demands. The descriptions of the groupings are shown in Table 2.

110 vehicles are generated in the network, placed at stations, and fully recharged as initialization. The maximum SOC is $100 \mathrm{~km}$ of those vehicles and the charging speed is $20 \mathrm{~km} /$ hour. The pattern of network and demand intensity is illustrated in Figure 7, where bubbles represent the locations of stations and the size of bubbles represents the intensity of demand.

Additional descriptive statistics of the original data set collected from the recent real operation environment are as follows. One vehicle would serve 5.17 trips per day on average; one station would process 10.16 trips per day on average. The station with maximum volume would serve 49 trips for a day and 10 trips in the peak hour. Features of group B present similar properties with the original data set. This study handles the demand set two times larger to test the capability of the proposed method.

Besides the transaction data, we also supplement the study with additional questionnaires to calibrate the 


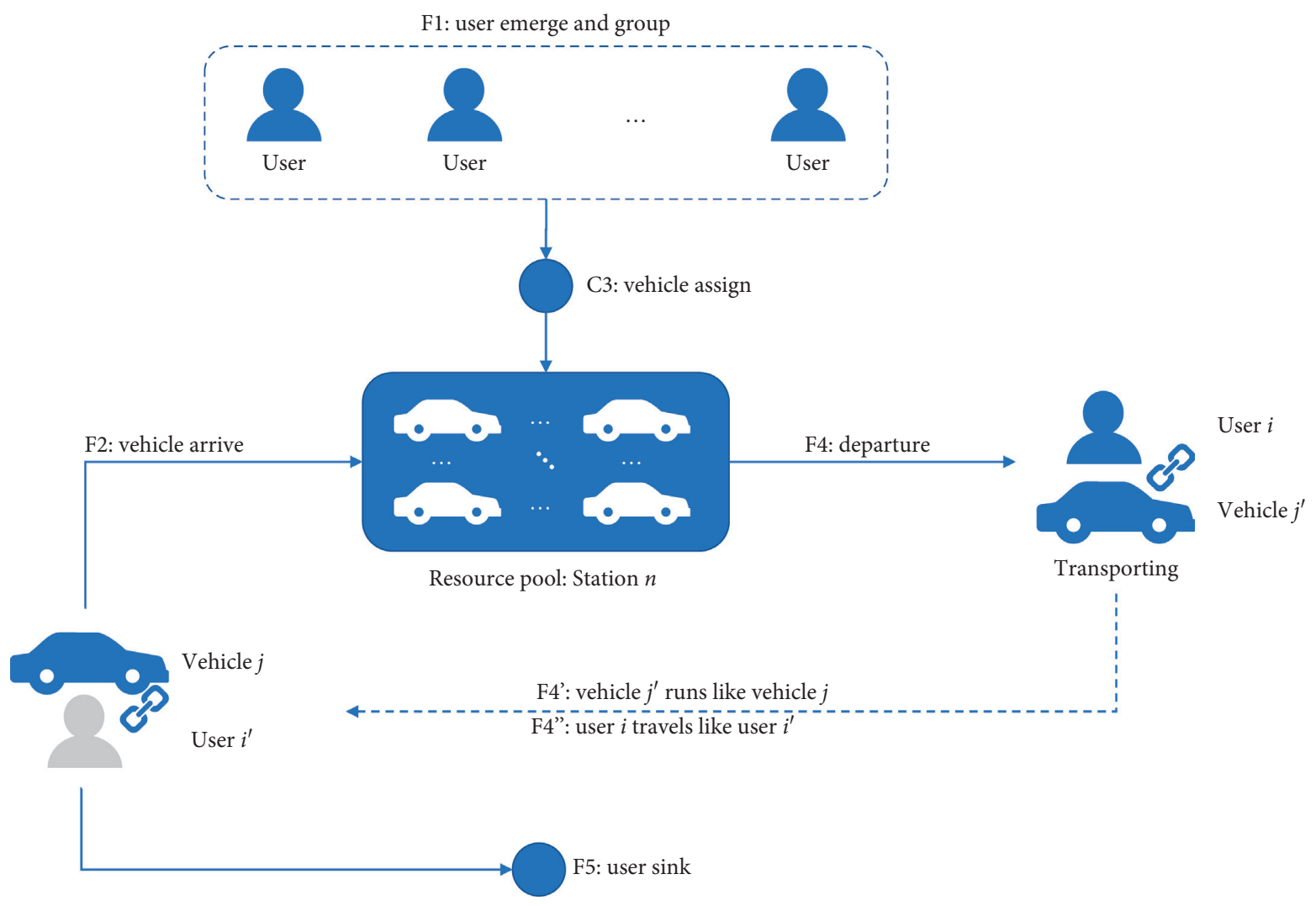

FIGURE 5: Events in short-term reservation mode.

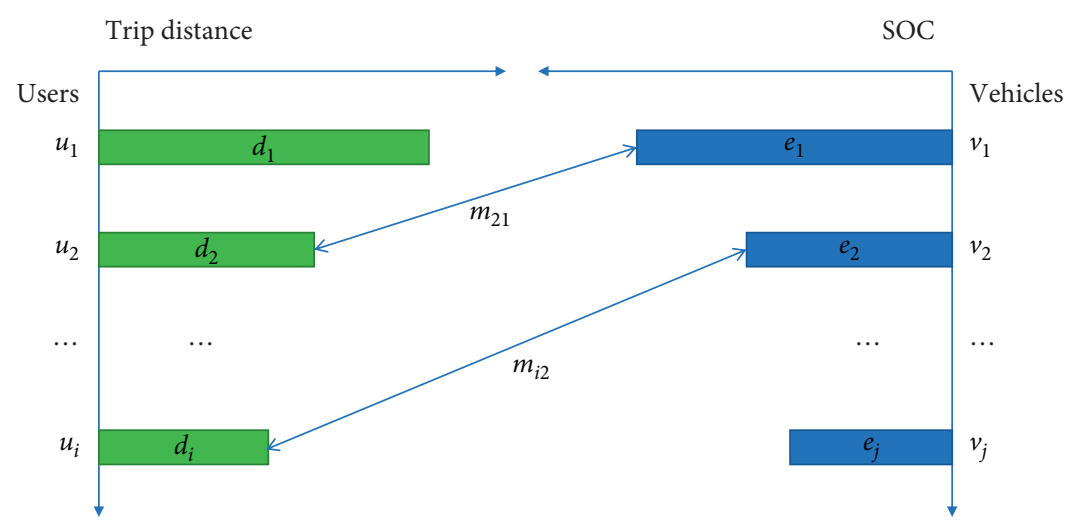

(a)

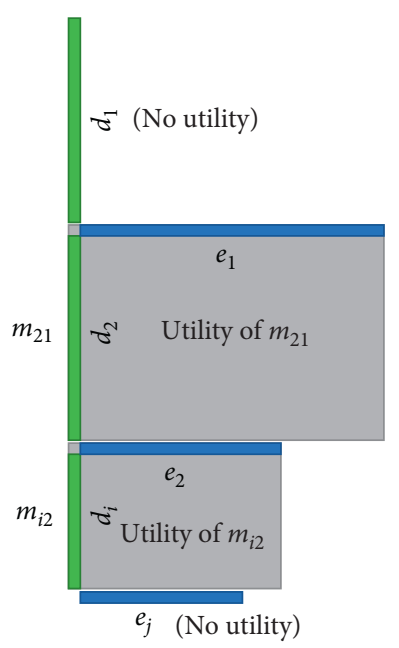

(b)

Figure 6: Matching between users and vehicles. (a) Visualization of matching. (b) Visualization of battery utility.

TABLE 2: Description of sample groups.

\begin{tabular}{lccc}
\hline Sample group & Number of requests & Size of demand & Demand scenario \\
\hline Group A & 286 & Half demand intensity & Undersaturated \\
Group B & 569 & Demand for one day & Balanced \\
Group C & 854 & 1.5 times demand & Oversaturated \\
Group D & 1138 & Double demand & Oversaturated \\
\hline
\end{tabular}




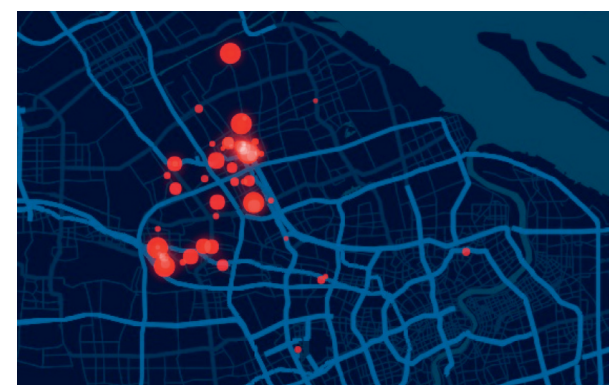

FIgURE 7: Network and demand intensity for case study.

probability that a user would choose the highest SOC or choose randomly, and the probability that a user would quit carsharing under different reservation times. We distributed the questionnaire within the users of this EVCS, and 218 valid samples were returned. Results and adopted values of the parameters are summarized in Table 3 . The survey also revealed users' attitudes towards battery redundancy that the average reserved SOC for users is $\varepsilon=10.2 \mathrm{~km}$ before they run out of battery. The revenue of the system is calculated by rental time, and the price is RMB 0.6 per minute.

4.2. Results. This section presents the results of testing the system performance under different schemes of instant access (IA) mode, short-term reservation mode with battery utility maximization (STR-BUM), and short-term reservation mode with battery utility and subsequent orders maximization (STR-BUSOM). To test the performance of each scheme, in this section we employ oversaturated demands of group C as inputs. Simulation of each scheme runs 10 times using the 10 copies of random inputs. Boxplots in Figure 8 show the results and compare the number of served and unserved demands across the three schemes.

Results show that the service satisfaction rate is around $70 \%$ of all schemes where STR-BUSOM served the most users (633 in average) and IA served the least (565.5 in average). About 288.5 users on average would not be served if IA was adopted, while 221 users would be rejected if STR-BUSOM was adopted. This phenomenon suggests that vehicle assignment by STR can serve more users than first-come-first-serve by IA mode.

The performance increment can be detected by the detailed indicators shown in Figure 9. First, if the total numbers of vehicles are the same, the utilization rate of vehicles should be higher so that more users could be served. Figure 9(a) shows that vehicles operated on STR-BUSOM can be utilized for a longer time in average than the other two. The higher utilization rate and more trips satisfied result in a higher amount of revenue (as shown in Figure 9(b)). The vehicles under STR-BUSOM are also driven for farther distances of more than $160 \mathrm{~km}$ in average, while in IA the vehicles traveled below $110 \mathrm{~km}$ in the simulation day (Figure 9(c)). From the perspective of users, the average travel trip is $28.06 \mathrm{~km}$ of STR-BUSOM, which is farther than $20.49 \mathrm{~km}$ of IA (Figure $7(\mathrm{~d})$ ).

Results of Figures 8 and 9 also imply the reasons accounting for the shortcomings of STR-BUM. Although more users are served than IA, STR-BUM shows a slight increment on vehicle occupied time, which is much lower than STR-BUSOM. It suggests that although STR-BUM matches more users with vehicles (satisfies more demands), vehicles would be idled subsequently at their next stations. However, STR-BUSOM effectively mobilizes vehicles into high-demand stations which are more likely to be utilized by continuous users, so the vehicle occupied time in average is longer. This feature can also be observed by detailed statistics in Figure 10. IA and STR-BUM present relatively similar distributions of travel distance per trip as shown in Figure 10(a), where STR-BUM presents a higher mean value. However, IA serves more trips with shorter distances, and STR-BUM assigns vehicles covering different ranges uniformly. STR-BUSOM is more prone to long distance trips. The statistics on idling time after vehicles arriving until next trips are also analyzed in Figure 10(b). STR-BUSOM reduces vehicle waiting time at stations, which confirms that it is prone to assign vehicles to users whose destinations are high-demand stations. Stations with high demands would be more likely to utilize vehicles to subsequent trips.

4.3. Simulation Experiments. To take deeper insights into the performances of IA and STR models, we designed further simulation experiments under different combinations of parameters. First, we inspect the system performance under different reservation times of $\tau=1,5,15,30,60$ minutes, and then we analyze the performance with the demand intensity of groups A to $D$.

The influence of short-term reservation time period $\tau$ is the most concerned parameter. This parameter influences the number of matchable incoming users and available vehicles and impacts user's acceptance of waiting. The result shows that the number of served requests firstly increases and then decreases of STR (as shown in Figure 11). The turning point happens at $\tau=15$ minutes. The number of served users of STRs drops lower than IA after adjusting the reservation time longer than 15 minutes (Figure 11(a)). The occupied time (Figure 11(b)) and traveled distance (Figure 11(c)) of vehicles on average present similar trends that first increase and then decrease. However, the average traveled distance of served users presents stable trends as shown in Figure 11(d).

We also extracted the results by setting users quit probability to zero. This is to test the contribution of adopting STR if persuading all users to fully accept STR as they accept IA. As shown in Figure 12, the number of served requests increases if the reservation time $\tau$ is enlarged. The increasing rate slows down and the trend levels out when $\tau$ increases (Figure 12(a)). The occupied time (Figure 12(b)) and traveled distance (Figure 12(c)) present similar increasing trends as well. Moreover, the average traveled distance of served users in Figure 12(d) also presents stable trends similar to Figure 11(d).

The performances of IA, STR-BUM, and STRBUSOM under different intensity of demands are also tested. As we employed different groups of trip requests samples, the performances of system under different 
TABle 3: Parameter calibration.

\begin{tabular}{lccc}
\hline Option & Number of samples & Parameter & Adopted value \\
\hline Choose vehicle with max SOC & 199 & $P$ & 0.913 \\
Choose vehicle randomly & 19 & $1-P$ & 0.087 \\
Quit if reservation time $\tau=1$ minute & 1 & $P=f(1)$ & 0.005 \\
Quit if reservation time $\tau=5$ minutes & 13 & $P=f(5)$ & 0.061 \\
Quit if reservation time $\tau=15$ minutes & 29 & $P=f(15)$ & 0.133 \\
Quit if reservation time $\tau=30$ minutes & 57 & $P=f(30)$ & 0.261 \\
Quit if reservation time $\tau=60$ minutes & 93 & $P=f(60)$ & 0.427 \\
\hline
\end{tabular}

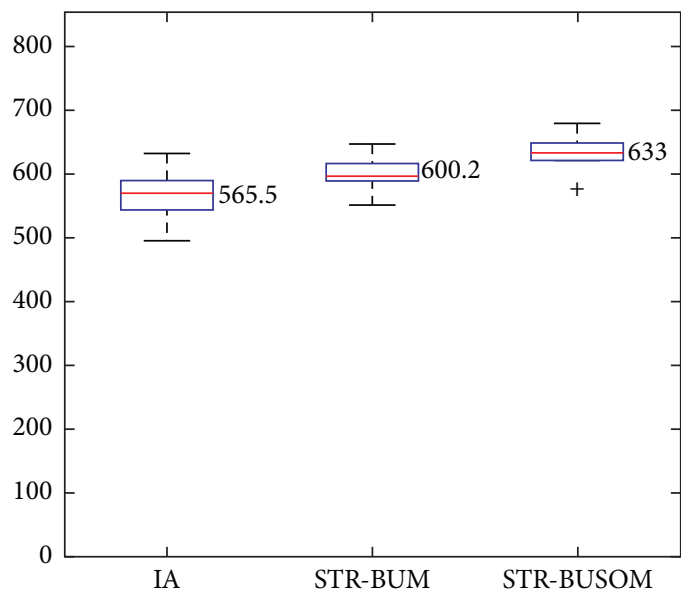

(a)

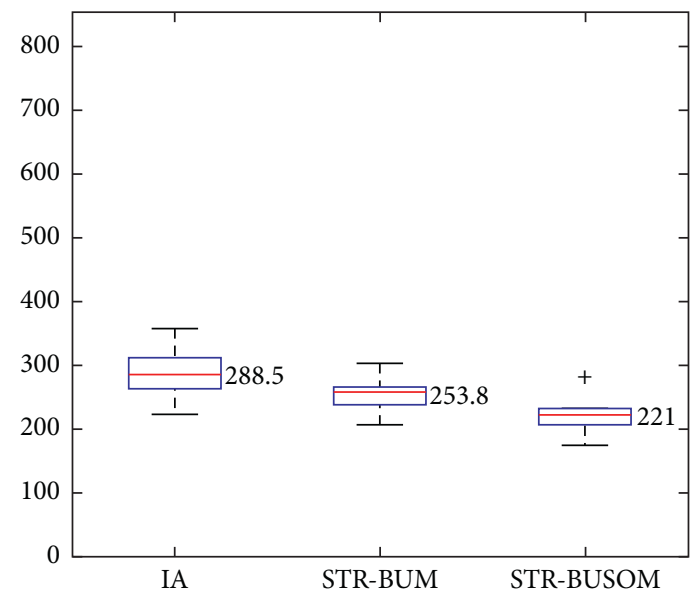

(b)

FIgURE 8: Comparisons on served and unserved demands. (a) Served demands. (b) Rejected demands.

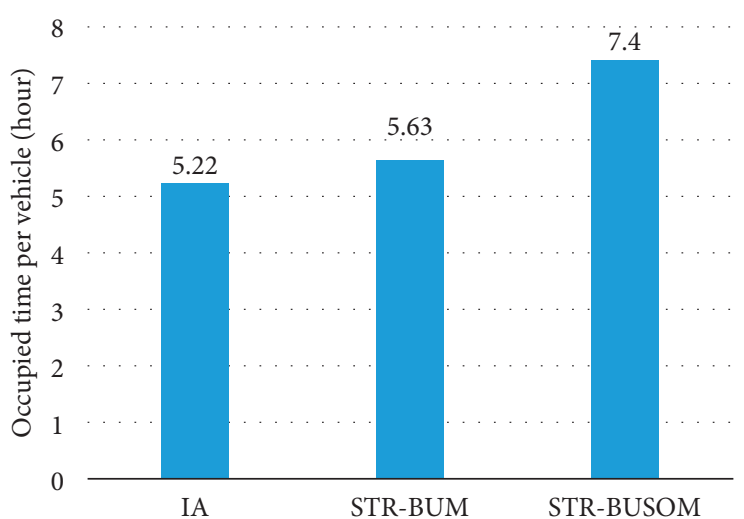

(a)

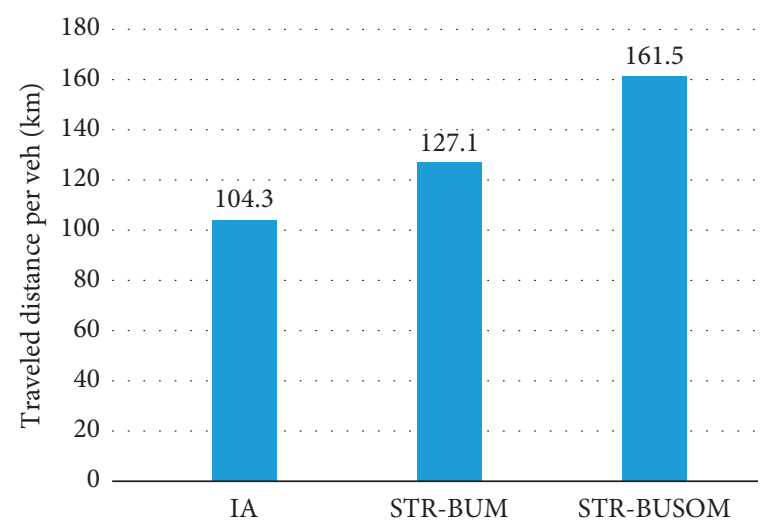

(c)

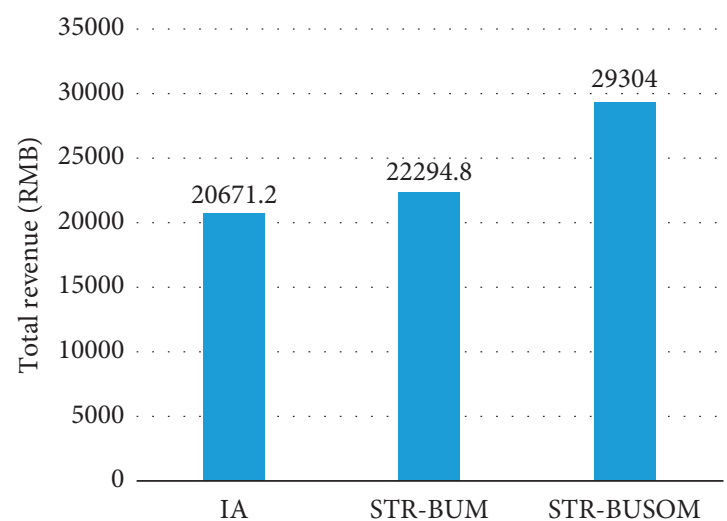

(b)

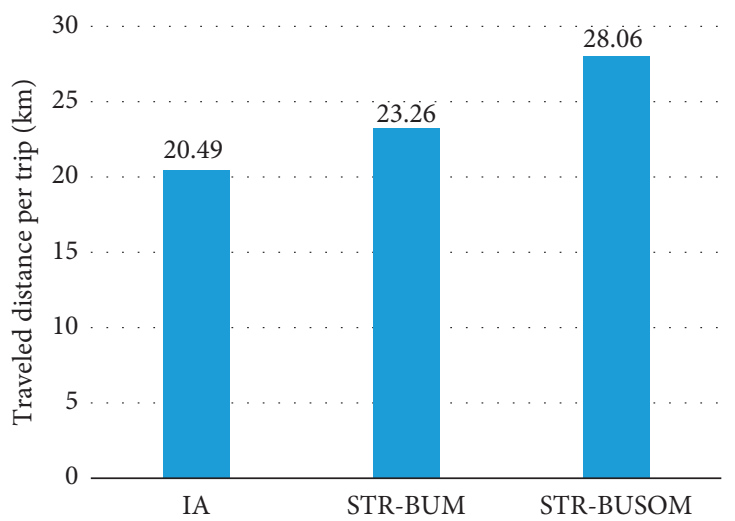

(d)

FIgURE 9: Comparisons on operation indicators. (a) Average occupied time per vehicle. (b) Total revenue. (c) Average traveled distance per vehicle. (d) Average traveled distance per trip. 


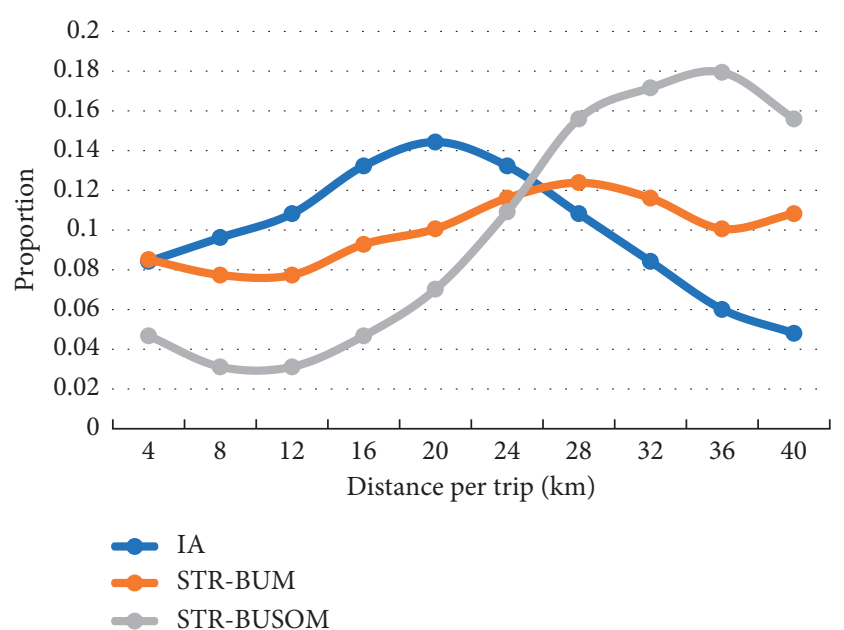

(a)

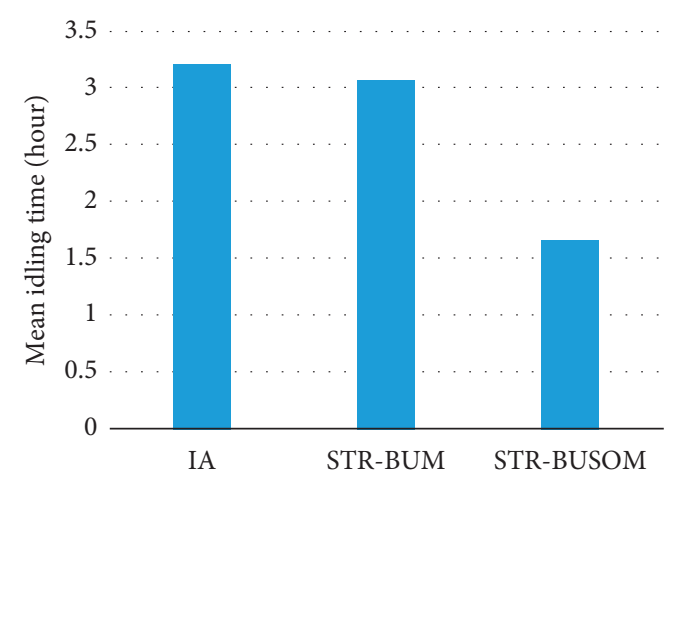

(b)

FIGURE 10: Detailed comparisons on performance of IA and STR. (a) Histogram of distance per trip. (b) Mean idling time.

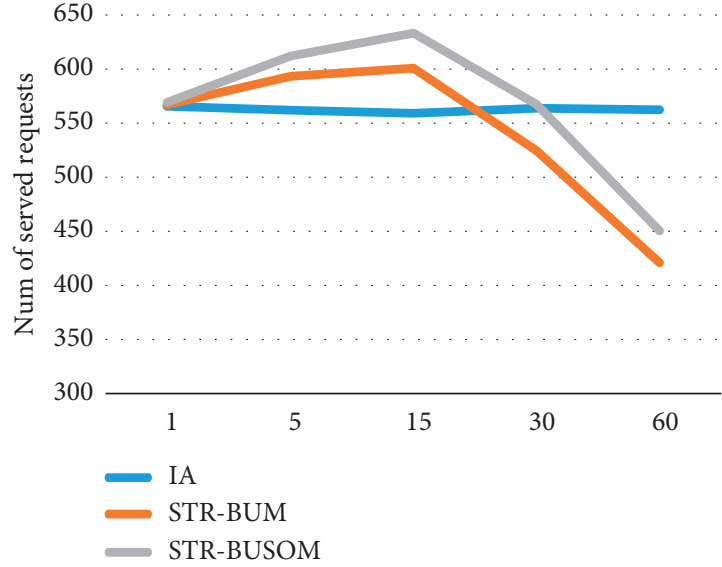

(a)

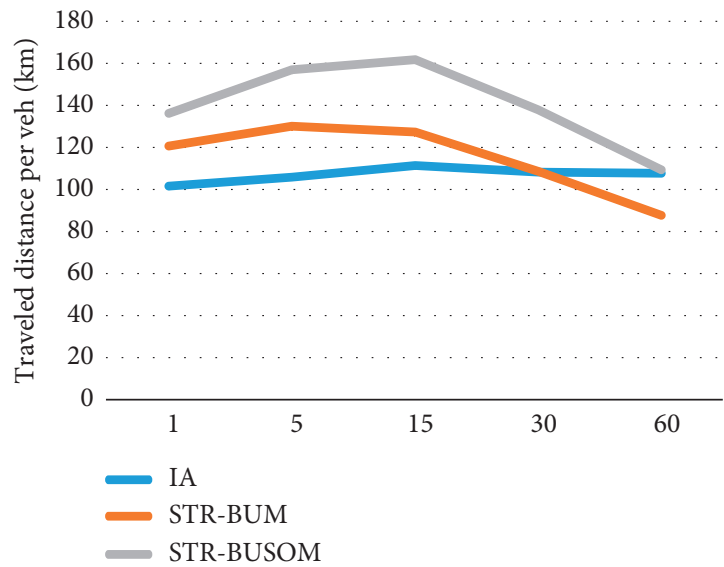

(c)

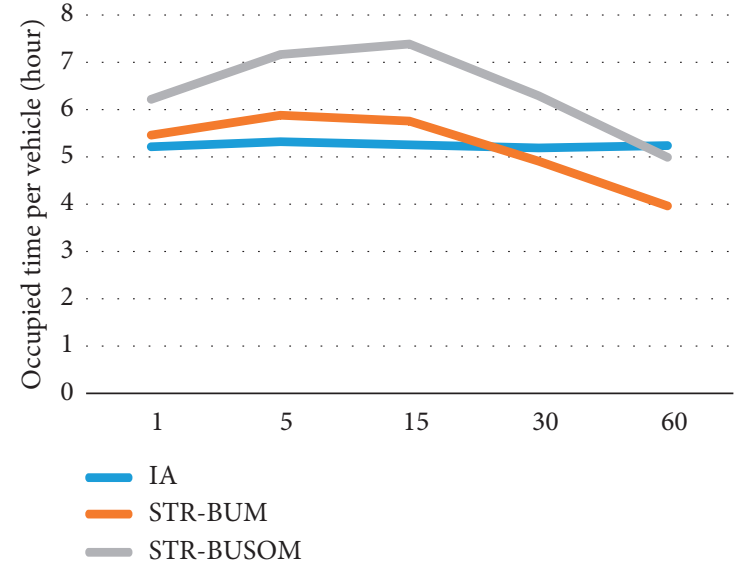

(b)

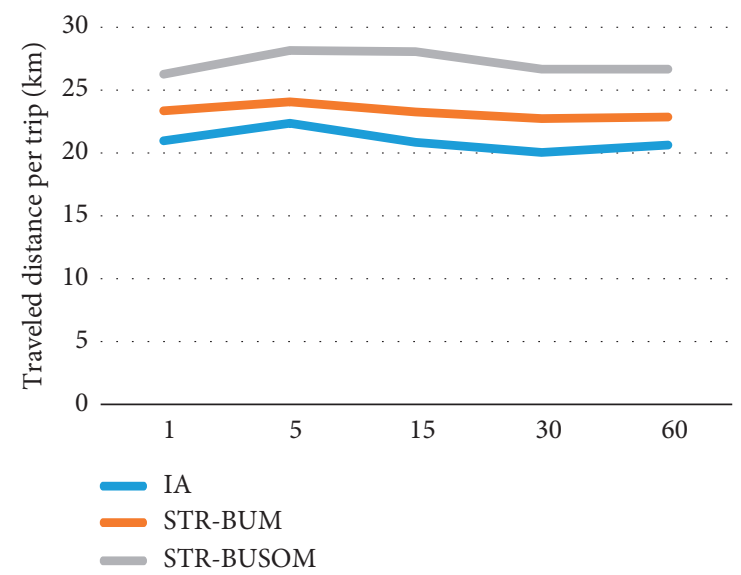

(d)

Figure 11: System performance for different values of $\tau$. (a) Number of served requests. (b) Average occupied time per vehicle. (c) Average traveled distance per vehicle. (d) Average traveled distance per trip.

vehicle selection schemes present different characteristics as shown in Figure 13. $\tau=15$ minutes is adopted in this analysis.
The grouping of demands shows features as follows. Group A represents light demands which is about half of group B; group B simulates the modest demand situation 


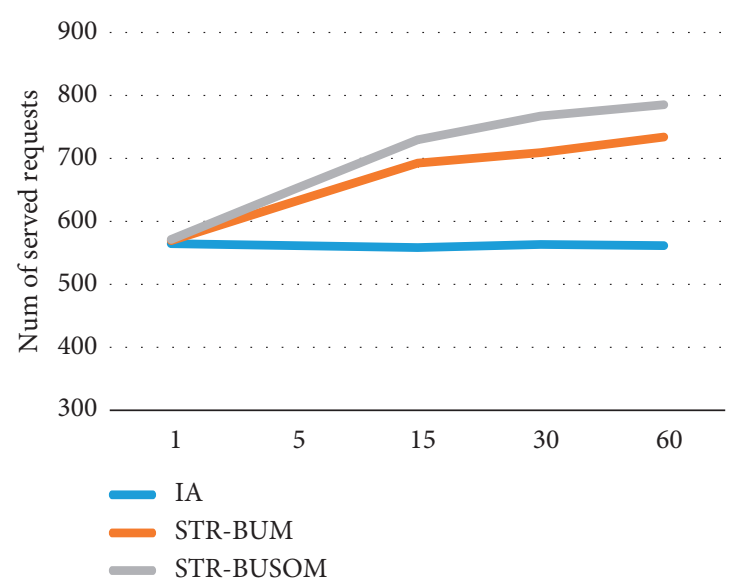

(a)

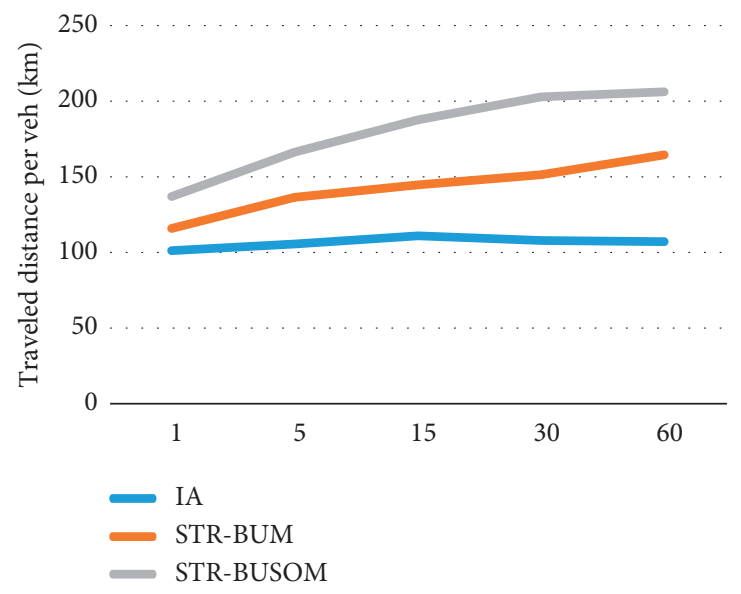

(c)

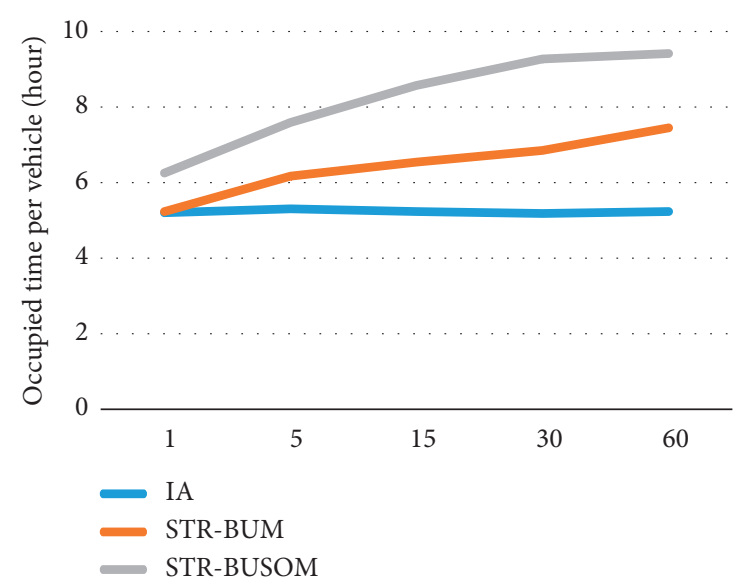

(b)

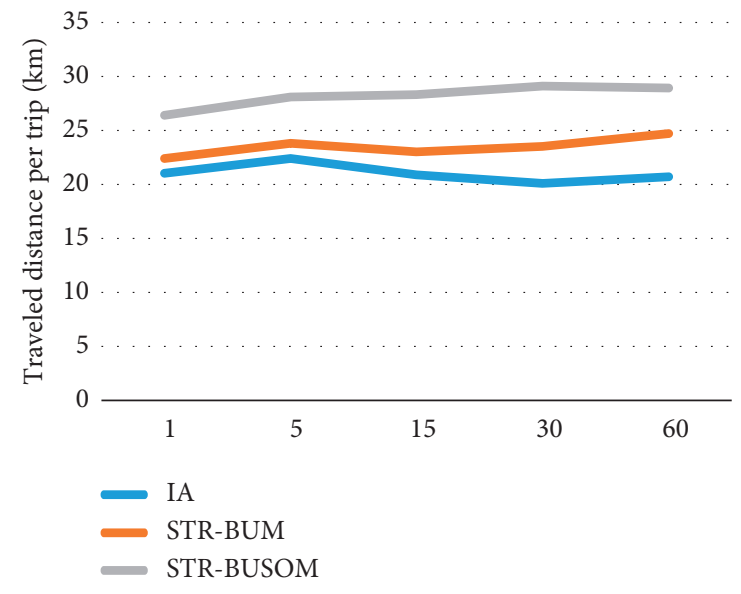

(d)

FIGURE 12: System potential if all users accept short-term reservation. (a) Number of served requests. (b) Average occupied time per vehicle. (c) Average traveled distance per vehicle. (d) Average traveled distance per trip.

which is close to the volume that is served in reality; group C represents the 1.5 times real demands; and group $D$ represents double demands and simulates the scenario of oversaturation. Each group scenario is tested 10 times with different random demands.

The numbers of served demand are similar among IA, STR-BUM, and STR-BUSOM at the scenario of group A (Figure 13(a)). The system performs slightly different if demand is under saturate. STRs performs slightly better than IA but not obviously. If increasing the input demands, the number of satisfied demands increases of STRs, while the increment of IA stops at group B, which also suggests the capacity of IA. The increments of STRs slow down and approximate to some capacities. The satisfaction ratios drop if increasing demands (Figure 13(b)). The occupied time of vehicles also increases if providing more demands but trends to level off (Figure 13(c)). The revenues under scenarios of group $\mathrm{C}$ and group seem similar and STRs could hardly earn more income if demands continue to increase, which implies the contribution of capacity increment made by STRs.
4.4. Findings and Discussions. The results from the case study and additional parameter experiments revealed some helpful findings for further study and industrial practice.

4.4.1. Short-Term Reservation Performs Better than Instant Access. STRs perform better than IA mode on both satisfied demands and system revenue. This result confirms that adopting short-term reservation and optimizing the vehicle assignment according to users' desired travel distance can satisfy more demand. This is probably because STRs can reduce the number of rejections on long distance requests. Users with demand with far driving distance can be served more, and users traveling nearby would be matched with vehicles with low SOC. On the other hand, vehicles with low SOC can be utilized by allocating them to users who drive nearby rather than letting them occupy vehicles with high SOC. Allocating vehicles to long distance driving users can earn more revenue by utilizing vehicles and shortening the idling 


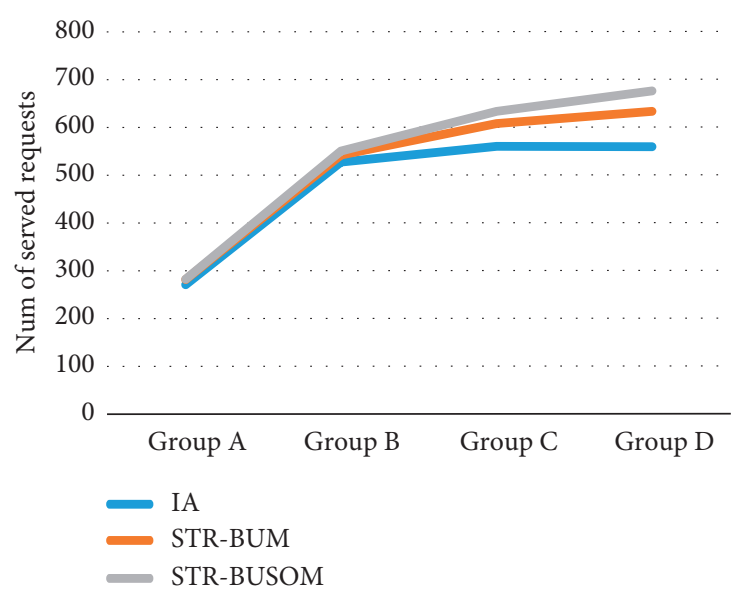

(a)

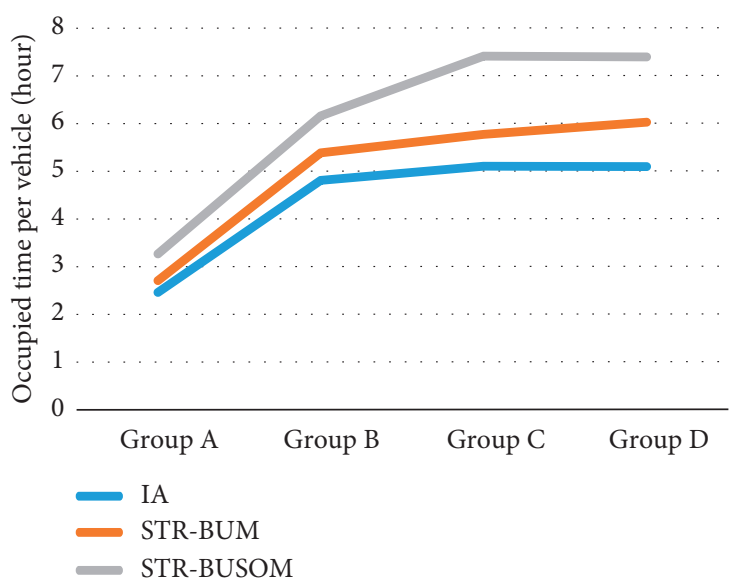

(c)

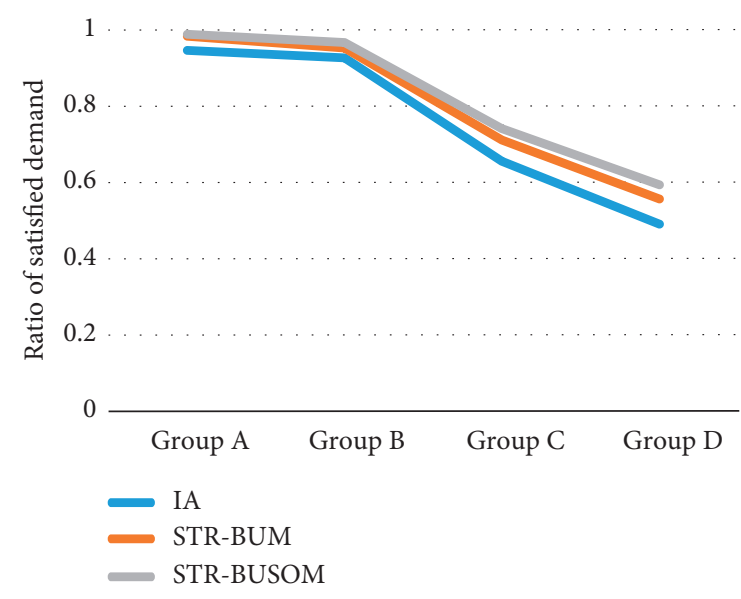

(b)

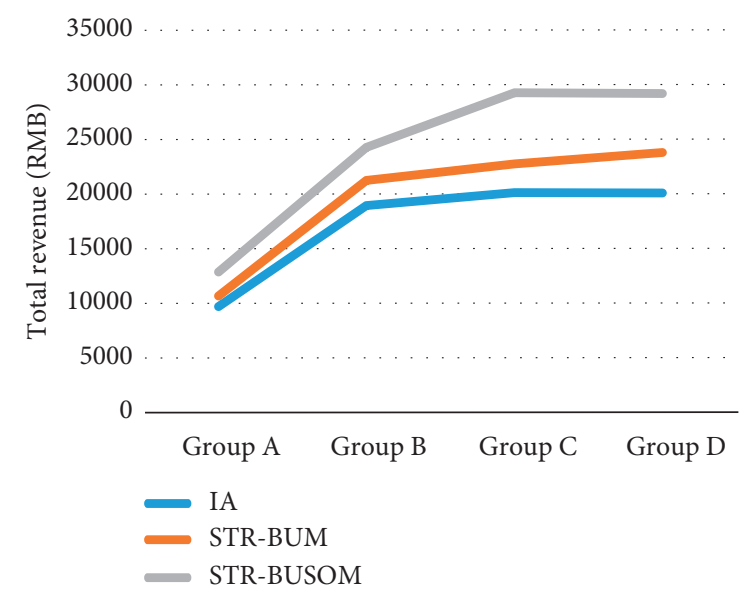

(d)

Figure 13: Performance and capacity under different demand intensity. (a) Number of served requests. (b) Ratio of satisfied demands to total demands. (c) Average occupied time per vehicle. (d) Total revenue.

time of them. This results in the increment of satisfied demands as well as the utilization rate of vehicles.

Note that the STR-BUSOM performs even better than STR-BUM. This is mainly because BUSOM allocates vehicles to users who drive to "hot spots," so that the vehicle could be soon utilized again. For IA system and STR-BUM system, it is possible for users to drive a vehicle to stations whose following demand is less, and vehicles would be idled for a longer time. However, the BUSOM is directional and selective to users and tends to have bias to serve users with farther distance requests preferentially, especially when the volume of demand is much higher than the vehicle supply capacity.

Since all existing one-way station-based EVCSs are instant access mode, we suggest CSOs to provide short-term reservation trials or service. This study reveals the benefit of short-term reservation; nevertheless, STR also enables vehicle relocation to fulfil more demands, which leads to better efficiency for the system operation. Note that the user's attitude to accept STR is quite important if they are accustomed to instant access service.

4.4.2. User Acceptability Impacts Short-Term Reservation Mode. The logic behind the short-term reservation is that the system could allocate vehicles more reasonably if more diverse requests from users are collected by extending the reservation time so that more requests would be served. The paradox is that users might not accept the longer reservation period and would quit the system or divert to other transportation modes.

In our study, we investigated user attitude towards shorttime reservation mode against instant access mode by additional questionnaire, and the experiments with simulation adopted the rejection probability of users to reshape the volume of demand. The result suggests that there is an inflection point through different widths of reservation time $\tau$. This implies that there would be an equilibrium between user acceptance towards STR and the width of reservation time.

CSOs are encouraged to motivate or persuade users to accept short-term reservation mode to better benefit from it. Short-term reservation is not difficult to be popularized since the user experience of it is similar to the current forms of online taxi-hailing, e-hailing, and ride-sharing, etc. Note that not all users would adapt to short-term reservation because some users with immediate trip requests would also emerge who are not willing to wait for a long time. In this 
study, the results suggest that setting the reservation time to 15 minutes would balance the user's needs and short-term resource allocation optimization.

4.4.3. Short-Term Reservation Provides Higher Capacity. Short-term reservation and instant access are similar for undersaturated demands, but different for oversaturated circumstances. We have discussed that IA serves fewer requests than STRs under similar parameters and scenarios due to higher efficiency of vehicle allocation of STRs. Experiments under different pressures of demand intensity suggest that when IA system touched the ceiling of capacity, STRs can serve even more requests, which implies that STRs present higher capacity to engage higher volume of demand.

The reason to account for this increment of capacity lies in the selectivity among the abundant demands that the system can choose users reversely if oversaturated. For FCFS like IA, the system can reach the highest capacity even though more demands emerge, but for the STRs the system dedicatedly allocates vehicles to users that might create more profits. STRs tend to allocate vehicles to users with far distance requirements and create more revenue, and especially for STR-BUSOM, the system will prefer to serve users who would drive to stations that would create more subsequent transactions. This is supported by the bodies of evidence that the utilization time and driving distance of vehicles of STRs are more than IA system.

\section{Conclusions}

This paper presents a simulation modeling process on both users selecting vehicles and optimal assigning vehicles to users for one-way station-based instant-access electric vehicle carsharing systems. The authors were aware of the fact that increasing the efficiency of matching the endurance running distances of available vehicles with the expected travel distances of users would serve more demand and create more profits. Users with requests on farther driving distance would be satisfied by allocating vehicles with higher SOC for them, and vehicles with lower battery endurance would be taken full advantage of by users who are traveling nearby, which avoids the circumstance that users traveling nearby occupy vehicles with higher SOC, leave short-range vehicles idling, and make other users with far distance demand being rejected.

The methodology of this research is developed from an agent-based discrete event simulation framework with firstcome-first-serve logic module and resource matching optimization module. The FCFS scheme is adopted to model the existing instant access carsharing service mode, and we proposed a vehicle assignment optimization model to better match the possible driving distances of vehicles with the expected driving distance of users. Moreover, we involved a criterion to allocate vehicles to users who drive vehicles to "hot spot" stations that create more revenue during the following operation.

Results of the case study reveal that the short-term reservation modes involving the vehicle assignment module perform better than the instant access mode, which serves more user requests and increases the revenue. Short-term reservation that considers the following transactions (STRBUSOM) performs even better than short-term reservation with battery utility maximization only.

This research encourages CSOs to enable short-term reservation for users to increase the capacity of their systems as well as incomes. Note that some parameters are not strictly calibrated and further discussed, e.g., whether users would mostly choose the vehicle with maximum SOC, or whether users would accept short-term reservation or insist on preferring the instant access mode. User behavior aspects should be further examined. This study also left the vehicle relocation alone and studied vehicle assignment only, which indicates that vehicle assignment promotes system performance. Further studies can combine vehicle relocation problems with vehicle assignment problem for not only short-term reservation but also long-term reservation carsharing systems.

\section{Notations}

\section{Symbol explanation}

$u_{i}: \quad \quad \quad$ User $i$, a tuple, $u_{i}=\left(O_{i}, D_{i}, t_{0 i}, t_{1 i}, d_{i}, m_{i}, r_{i}, s_{i}\right)$

$U: \quad$ A set of users, $U=\left\{u_{1}, u_{2}, \ldots, u_{i}, \ldots, u_{|U|}\right\},|U|$ denotes the number of users in set $U$

$v_{j}: \quad \quad \quad$ Vehicle $j$, a tuple, $v_{j}=\left(e_{j}, p_{j}, t_{A j}, r_{O j}\right)$

$V: \quad \quad$ A set of vehicles, $V=\left\{v_{1}, v_{2}, \ldots, v_{j}, \ldots, v_{|V|}\right\}$,

$|V|$ denotes the number of vehicles in set $V$, where $V_{I}$ and $V_{R}$ are for idling vehicles and running vehicles, respectively

$m_{i j}$ : The matching between user and vehicle, $m_{i j}=\left(u_{i}, v_{j}\right)$

$N$ : $\quad$ A set of stations, where $n \in N$ denotes a station and $V_{n}$ denotes the set of vehicles parking at station $n$

$O_{i}: \quad$ The origin station of $u_{i}$

$D_{i}: \quad$ The destination station of $u_{i}$

$t_{0 i}: \quad$ The departure time of $u_{i}$

$t_{1 i}: \quad$ The arrival time of $u_{i}$

$d_{i}$ : The requested trip distance of $u_{i}, \mathrm{~km}$

$m_{i}: \quad$ A $0-1$ sign, $m_{i}=1$ if user $i$ is matched with a vehicle that can satisfy the user's trip; otherwise $=0$

$r_{i}$ : A $0-1$ sign, whether the user is occupying and driving a car on road; $r_{i}=1$ if driving; otherwise $=0$

$s_{i}$ : $\quad$ A $0-1$ sign, whether the user is served; $s_{i}=1$ if served; otherwise $=0$

$e_{j}$ : The instant state of charge (SOC) of vehicle $j$, defined by the current travel distance endurance, $\mathrm{km}$

$e_{M}$ : $\quad$ Maximum endurance distance when the charging is finished, $\mathrm{km}$

$\varepsilon$ : $\quad$ Reserved distance in case that the vehicle is out of energy

$p_{j}: \quad$ The position of $v_{j}$ by the latest station where $v_{j}$ is parking

$t_{A j}: \quad$ The arrival time at $p_{j} ; t_{A j}=0$ for initialization 


$$
\begin{aligned}
& r_{\mathrm{O} j} \text { : The occupancy of the vehicle and } r_{\mathrm{O} j}=1 \text { if } v_{j} \text { is } \\
& \text { occupied by a user; otherwise } r_{O j}=0 \\
& V_{A i}: \quad \text { A set of available vehicles for user } i \text { to satisfy the } \\
& \text { trip distance } \\
& \operatorname{rand}\left(V_{A i}\right): \text { A function which randomly returns a } v_{j} \text { from } \\
& V_{A i} \\
& t_{M}: \quad \text { A very short time interval } \\
& \tau: \quad \text { Time interval to gather a batch of requests, i.e., } \\
& \text { every } \tau \text { s, i.e., the } 0 \mathrm{~s}, \tau \mathrm{s}, 2 \tau \mathrm{s}, 3 \tau \mathrm{s}, \ldots, k \tau \mathrm{s}, \ldots K \tau \mathrm{s} \\
& \text { the matching event is activated } \\
& U_{k n}: \quad \text { The set of users at station } n \text { during time period } k \\
& R: \quad \text { Charging rate to extend the running distance, } \\
& \mathrm{km} / \mathrm{s} \\
& W_{n}: \quad \text { Weight of importance of station } n \\
& x_{i j} \text { : } \quad 0-1 \text { variables, mark that the vehicle } j \text { is assigned } \\
& \text { to user } i \text { if } x_{i j}=1 \text {; otherwise } 0 \text {. }
\end{aligned}
$$

\section{Data Availability}

The original data that supports this paper can be accessed at the website on https://drive.google.com/drive/folders/ 1qZOpGqHCG1dCtUlnMUlFRnPjuh5lp5sZ.

\section{Conflicts of Interest}

The authors declare that there are no conflicts of interest regarding the publication of this paper.

\section{Acknowledgments}

The authors would like to extend their thanks to the support provided by the the China National Postdoctoral Program for Innovative Talents (grantNo. BX20190241), the Key Research and Development Program of Shaanxi Province, China (Grant nos. 2019ZDLGY15-04-02, 2018ZDCXL-GY05-07-02, 2019GY-083, and 2019GY-059), the National Natural Science Foundation of China (Grant no. 71901040), the Key Science and Technology Program of Shaanxi Province, China (Grant nos. 2019JQ-442), the Fundamental Research Funds for the Central Universities (Grant no. 300102329502), and the China Postdoctoral Science Foundation (project No. 2020M671223).

\section{References}

[1] T. F. Luna, M. Uriona-Maldonado, M. E. Silva, and C. R. Vaz, "The influence of e-carsharing schemes on electric vehicle adoption and carbon emissions: an emerging economy study," Transportation Research Part D: Transport and Environment, vol. 79, Article ID 102226, 2020.

[2] S. Shaheen, E. Martin, and H. Totte, "Zero-emission vehicle exposure within U.S. carsharing fleets and impacts on sentiment toward electric-drive vehicles," Transport Policy, vol. 85, pp. A23-A32, 2020.

[3] S. Hu, P. Chen, H. Lin, C. Xie, and X. Chen, "Promoting carsharing attractiveness and efficiency: an exploratory analysis," Transportation Research Part D: Transport and Environment, vol. 65, pp. 229-243, 2018.

[4] M. Barth and S. Shaheen, "Shared-use vehicle systems: framework for classifying carsharing, station cars, and combined approaches," Journal of the Transportation Research Board, vol. 1791, pp. 105-112, 2002.

[5] E. M. Cepolina, A. Farina, C. Holloway, and N. Tyler, "Innovative strategies for urban car-sharing systems and a simulator to assess their performance," Transportation Planning and Technology, vol. 38, no. 4, pp. 375-391, 2015.

[6] L. Wang, Y. Zhong, and W. Ma, "GPS-data-driven dynamic destination prediction for on-demand one-way carsharing system," IET Intelligent Transport Systems, vol. 12, no. 10, pp. 1291-1299, 2018.

[7] B. Boyac1 and K. G. Zografos, "Investigating the effect of temporal and spatial flexibility on the performance of oneway electric carsharing systems," Transportation Research Part B: Methodological, vol. 129, pp. 244-272, 2019.

[8] C. A. Folkestad, N. Hansen, K. Fagerholt, H. Andersson, and G. Pantuso, "Optimal charging and repositioning of electric vehicles in a free-floating carsharing system," Computers \& Operations Research, vol. 113, Article ID 104771, 2020.

[9] G. Remane, R. C. Nickerson, A. Hanelt, J. Tesch, and L. M. Kolbe, "A taxonomy of carsharing business models," in Proceedings of the at the 37th International Conference on Information Systems, Dublin, Ireland, 2016.

[10] Q. Kang, J. Wang, M. Zhou, and A. C. Ammari, "Centralized charging strategy and scheduling algorithm for electric vehicles under a battery swapping scenario," IEEE Transactions on Intelligent Transportation Systems, vol. 17, no. 3, pp. 659-669, 2016.

[11] Q. Kang, S. Feng, M. Zhou, A. C. Ammari, and K. Sedraoui, "Optimal load scheduling of plug-in hybrid electric vehicles via weight-aggregation multi-objective evolutionary algorithms," IEEE Transactions on Intelligent Transportation Systems, vol. 18, no. 9, pp. 2557-2568, 2017.

[12] C. Luo, Z. Shen, S. Evangelou, G. Xiong, and F.-Y. Wang, "The combination of two control strategies for series hybrid electric vehicles," IEEE/CAA Journal of Automatica Sinica, vol. 6, no. 2, pp. 596-608, 2019.

[13] X. Kong, M. Li, T. Tang, K. Tian, L. Moreira-Matias, and F. Xia, "Shared subway shuttle bus route planning based on transport data analytics," IEEE Transactions on Automation Science and Engineering, vol. 15, no. 4, pp. 1507-1520, 2018.

[14] R. Xie, W. Wei, Q. Wu, T. Ding, and S. Mei, "Optimal service pricing and charging scheduling of an electric vehicle sharing system," IEEE Transactions on Vehicular Technology, vol. 69, no. 1, pp. 78-89, 2020.

[15] S. Hu, P. Chen, F. Xin, and C. Xie, "Exploring the effect of battery capacity on electric vehicle sharing programs using a simulation approach," Transportation Research Part D: Transport and Environment, vol. 77, pp. 164-177, 2019.

[16] S. Hu, H. Lin, K. Xie, X. Chen, and H. Shi, "Modeling users' vehicles selection behavior in the urban carsharing program," in Proceedings of the 21st International Conference on Intelligent Transportation Systems (ITSC), pp. 1546-1551, 2018.

[17] L. Noel, G. Zarazua De Rubens, B. K. Sovacool, and J. Kester, "Fear and loathing of electric vehicles: the reactionary rhetoric of range anxiety," Energy Research \& Social Science, vol. 48, pp. 96-107, 2019.

[18] C. Xie, T.-G. Wang, X. Pu, and A. Karoonsoontawong, "Pathconstrained traffic assignment: modeling and computing network impacts of stochastic range anxiety," Transportation Research Part B: Methodological, vol. 103, pp. 136-157, 2017.

[19] G. Brandstätter, M. Kahr, and M. Leitner, "Determining optimal locations for charging stations of electric car-sharing systems under stochastic demand," Transportation Research Part B: Methodological, vol. 104, pp. 17-35, 2017. 
[20] A. Deza, K. Huang, and M. R. Metel, "Charging station optimization for balanced electric car sharing," Discrete Applied Mathematics, 2020.

[21] M. Xu, H. Yang, and S. Wang, "Mitigate the range anxiety: siting battery charging stations for electric vehicle drivers," Transportation Research Part C: Emerging Technologies, vol. 114, pp. 164-188, 2020.

[22] S. Pelletier, O. Jabali, and G. Laporte, "The electric vehicle routing problem with energy consumption uncertainty," Transportation Research Part B: Methodological, vol. 126, pp. 225-255, 2019.

[23] A. Almouhanna, C. L. Quintero-Araujo, J. Panadero, A. A. Juan, B. Khosravi, and D. Ouelhadj, "The location routing problem using electric vehicles with constrained distance," Computers \& Operations Research, vol. 115, Article ID 104864, 2020.

[24] D. Zhang, Y. Liu, and S. He, "Vehicle assignment and relays for one-way electric car-sharing systems," Transportation Research Part B: Methodological, vol. 120, pp. 125-146, 2019.

[25] A. Kek, R. Cheu, and M. Chor, "Relocation simulation model for multiple-station shared-use vehicle systems," Transportation Research Record: Journal of the Transportation Research Board, vol. 1986, no. 1, pp. 81-88, 2006.

[26] M. Barth and M. Todd, "Simulation model performance analysis of a multiple station shared vehicle system," Transportation Research Part C: Emerging Technologies, vol. 7, no. 4, pp. 237-259, 1999.

[27] G. Alfian, J. Rhee, and B. Yoon, "A relocation simulation model for one-way carsharing service," in Proceedings of the Industrial Technology (ICIT), Busan, Korea, 2014.

[28] L. Li, D. Lin, T. Pantelidis, J. Chow, and S. E. Jabari, “An agent-based simulation for shared automated electric vehicles with vehicle relocation," in Proceedings of the IEEE Intelligent Transportation Systems Conference (ITSC), pp. 3308-3313, Rhodes, Greece, 2019.

[29] M. Bruglieri, F. Pezzella, and O. Pisacane, "A two-phase optimization method for a multiobjective vehicle relocation problem in electric carsharing systems," Journal of Combinatorial Optimization, vol. 36, no. 1, pp. 162-193, 2018.

[30] X. Li, J. Ma, J. Cui, A. Ghiasi, and F. Zhou, "Design framework of large-scale one-way electric vehicle sharing systems: a continuum approximation model," Transportation Research Part B: Methodological, vol. 88, pp. 21-45, 2016.

[31] G. Brandstätter, C. Gambella, M. Leitner et al., "Overview of optimization problems in electric car-sharing system design and management," Dynamic Perspectives on Managerial Decision Making, pp. 441-471, Springer, Berlin, Germany, 2016.

[32] B. Boyac1, K. G. Zografos, and N. Geroliminis, "An optimization framework for the development of efficient one-way car-sharing systems," European Journal of Operational Research, vol. 240, pp. 718-733, 2015.

[33] W. Fan, "Management of dynamic vehicle allocation for carsharing systems: stochastic programming approach," Transportation Research Record: Journal of the Transportation Research Board, vol. 2359, no. 1, pp. 51-58, 2013.

[34] M. Nourinejad, S. Zhu, S. Bahrami, and M. J. Roorda, "Vehicle relocation and staff rebalancing in one-way carsharing systems," Transportation Research Part E: Logistics and Transportation Review, vol. 81, pp. 98-113, 2015.

[35] M. Repoux, M. Kaspi, B. Boyac1, and N. Geroliminis, "Dynamic prediction-based relocation policies in one-way station-based carsharing systems with complete journey reservations," Transportation Research Part B: Methodological, vol. 130, pp. 82-104, 2019.

[36] G. Molnar and G. H. D. A. Correia, "Long-term vehicle reservations in one-way free-floating carsharing systems: a variable quality of service model," Transportation Research Part C: Emerging Technologies, vol. 98, pp. 298-322, 2019.

[37] L. Wang, H. Zhong, W. Ma, Y. Zhong, and L. Wang, "Multisource data-driven prediction for the dynamic pickup demand of one-way carsharing systems," Transportmetrica B: Transport Dynamics, vol. 8, no. 1, pp. 90-107, 2020.

[38] D. Yu, Z. Li, Q. Zhong, Y. Ai, and W. Chen, "Demand management of station-based car sharing system based on deep learning forecasting," Journal of Advanced Transportation, vol. 2020, Article ID 8935857, 15 pages, 2020.

[39] B. Boyac1, K. G. Zografos, and N. Geroliminis, "An integrated optimization-simulation framework for vehicle and personnel relocations of electric carsharing systems with reservations," Transportation Research Part B: Methodological, vol. 95, pp. 214-237, 2017.

[40] M. Repoux, B. Boyaci, and N. Geroliminis, "Simulation and optimization of one-way car-sharing systems with variant relocation policies," in Proceedings of the 94th Annual meeting of Transportation Research Board, Washington, DC, USA, 2015.

[41] A. G. H. Kek, R. L. Cheu, Q. Meng, and C. H. Fung, "A decision support system for vehicle relocation operations in carsharing systems," Transportation Research Part E: Logistics and Transportation Review, vol. 45, no. 1, pp. 149-158, 2009.

[42] L. Wang, Q. Liu, and W. Ma, "Optimization of dynamic relocation operations for one-way electric carsharing systems," Transportation Research Part C: Emerging Technologies, vol. 101, pp. 55-69, 2019.

[43] W. Ma, L. Wang, and L. Li, "Vehicle relocation triggering thresholds determination in electric carsharing system under stochastic demand," Journal of Advanced Transportation, vol. 15, 2018. 